\title{
HÖLDER CONTINUOUS SOLUTIONS TO MONGE-AMPÈRE EQUATIONS
}

\author{
JEAN-PIERRE DEMAILLY, SŁAWOMIR DINEW, VINCENT GUEDJ, PHAM HOANG HIEP, \\ SEAWOMIR KOŁODZIEJ AND AHMED ZERIAHI
}

\begin{abstract}
Let $(X, \omega)$ be a compact Kähler manifold. We obtain uniform Hölder regularity for solutions to the complex Monge-Ampère equation on $X$ with $L^{p}$ right hand side, $p>1$. The same regularity is furthermore proved on the ample locus in any big cohomology class. We also study the range $\operatorname{MAH}(X, \omega)$ of the complex Monge-Ampère operator acting on $\omega$ plurisubharmonic Hölder continuous functions. We show that this set is convex, by sharpening Kołodziej's result that measures with $L^{p}$-density belong to $\operatorname{MAH}(X, \omega)$ and proving that $\operatorname{MAH}(X, \omega)$ has the " $L^{p}$-property", $p>1$. We also describe accurately the symmetric measures it contains.
\end{abstract}

\section{INTRODUCTION}

Let $(X, \omega)$ be a compact $n$-dimensional Kähler manifold. Let also $\Theta=\{\theta\} \in H^{1,1}(X, \mathbb{R})$ be a given cohomology class on $X$. In the note we consider two different cases of interest:

(1) $\Theta$ is a Kähler class, i.e. there exists a Kähler form which represents $\Theta$. In this case we assume without loss of generality that $\omega \in \Theta$;

(2) $\Theta$ is a big cohomology class, which means that there exists a (possibly singular) closed $(1,1)$ current $T$ representing $\Theta$ such that $T$ is strictly positive i.e. $T \geq \varepsilon_{0} \omega$ for some constant $\varepsilon_{0}>0$.

The study of complex Monge-Ampère equations on compact Kähler manifolds with a Kähler background metric has a long history and many spectacular results have appeared during the years. The big cohomology class setting, on the other hand, was initiated recently in [BEGZ]. This is the most general setting where a meaningful (and nontrivial) theory can be developed. Of course it covers the Kähler class setting as a particular case, but since the latter is more classical and certain technicalities can be avoided we have decided to treat both cases separately.

We deal with the Kähler setting first. We study the range of the (normalized) complex Monge-Ampère operator

$$
\operatorname{MA}(u):=\frac{1}{V_{\omega}}\left(\omega+d d^{c} u\right)^{n}, \quad V_{\omega}:=\operatorname{Vol}_{\omega}(X)=\int_{X} \omega^{n},
$$

acting on $\omega$-plurisubharmonic ( $\omega$-psh for short) Hölder-continuous functions $u$. Here, as usual $d=\partial+\bar{\partial}$ and $d^{c}:=\frac{1}{2 i \pi}(\partial-\bar{\partial})$, and $V_{\omega}$ denotes the volume of the cohomology class $\{\omega\}$, so that $M A(u)$ is a probability measure.

This problem is motivated by the study of canonical metrics on mildly singular varieties: their potentials are solutions to degenerate complex Monge-Ampère equations for which Hölder continuity is the best global regularity one can expect. Furthermore even such weak regularity does imply estimates for the "metric" $\omega+d d^{c} u$ which might be relevant for the study of the limiting behavior of the Kähler-Ricci flow. We refer the reader to [ST1, ST2, ST3, SW, EGZ1, GKZ, K3, KT, BCHM, BEGZ, To, TZ, SW] for further geometrical motivations and references.

Date: October 8, 2018.

2000 Mathematics Subject Classification. 32U05, 32U40, 53C55.

Key words and phrases. Monge-Ampère operator, Kähler manifold, pluripotential theory, Hölder continuity. 
We let $\operatorname{PSH}(X, \omega)$ denote the set of $\omega$-psh functions: these are defined as being locally equal to the sum of a plurisubharmonic and a smooth function and any such function $u$ additionally satisfies the inequality $\omega+d d^{c} u \geq 0$ in the weak sense of currents.

We let $\operatorname{Hölder}(X, \mathbb{R})$ denote the set of real valued Hölder-continuous functions on $X$. Our goal is thus to understand the range

$$
\operatorname{MAH}(X, \omega):=\operatorname{MA}(\operatorname{PSH}(X, \omega) \cap \operatorname{Hölder}(X, \mathbb{R})) .
$$

A result of fifth named author [K3] (see [EGZ1] and [Di] for refinements in particular cases) asserts that a probability measure $\mu=f \omega^{n}$ which is absolutely continuous with respect to the Lebesgue measure belongs to $\operatorname{MAH}(X, \omega)$ if it has density $f \in L^{p}$ for some $p>1$. Note that a Monge-Ampère potential $u \in P S H(X, \omega)$ such that $M A(u)=\mu$ is unique, up to an additive constant.

The proof in [K3] does not give any information on the Hölder exponent of the Monge-Ampère potential. We combine here the methods of [K3] and the regularization techniques of the first named author [D1, D4] to establish the following result:

Theorem A. Let $\mu=f \omega^{n}=M A(u)$ be a probability measure absolutely continuous with respect to Lebesgue measure, with density $f \in L^{p}, p>1$. Then $u$ is Hölder-continuous with exponent $\alpha$ arbitrarily close to $2 /(1+n q)$, where $q$ denotes the conjugate exponent of $p$.

It is a slightly better exponent than the one obtained in some special cases in [EGZ1] and [Di]. It is also asymptotically optimal (see [Pl] and [GKZ] for some local counterexamples which are easily adjustable to the compact setting). The proof uses a subtle regularization result of $[\mathrm{D} 1, \mathrm{D} 4]$, as in [Di] and [BD]. The extra tool that allows us to remove symmetry/curvature constraints is the Kiselman minimum principle coupled with Demailly's method of attenuating singularities (the Kiselman-Legendre transform) from [D4].

By keeping track of the Hölder constant together with the exponent one can in fact obtain uniform estimates provided suitable control on the global geometry is assumed. More precisely if we assume uniformly bounded geometry (this notion will be explained in the Preliminaries) the following holds:

Theorem A*. Let $\left(X_{s}, \omega_{s}\right)$ be a family of compact Kähler manifolds with uniformly bounded geometry. Consider the Monge-Ampère equations

$$
\left(\omega_{s}+d d^{c} u_{s}\right)^{n}=f_{s} \omega_{s}^{n}, \quad \sup _{X_{s}} u_{s}=0 .
$$

If $\|f\|_{L^{p}\left(\omega_{s}^{n}\right)} \leq C$ are uniformly bounded then the solutions $u_{s}$ are uniformly Hölder continuous for any exponent $\alpha<2 /(n q+1)$ and the Hölder constant is uniformly controlled by $C$ and the constants from the definition of the uniformly bounded geometry.

We furthermore believe that additional technical improvements of our arguments may lead to analogous statements in the case of classes which are merely semi-positive and big (see [BGZ] for a definition and further developments).

A satisfactory description of $\operatorname{MAH}(X, \omega)$ is yet to be found. We nevertheless establish a technically involved characterization (Theorem 4.3) that allows us to derive several useful consequences, for example we show:

Theorem B. The set $\operatorname{MAH}(X, \omega)$ has the $L^{p}$-property: if $\mu \in \operatorname{MAH}(X, \omega)$ and $0 \leq f \in L^{p}(\mu)$ with $p>1$ and $\int_{X} f d \mu=1$, then $f \mu \in \operatorname{MAH}(X, \omega)$.

In particular the set $\mathrm{MAH}(X, \omega)$ is convex.

It has been recently proved by Dinh-Nguyen-Sibony [DNS] (see also [Ber] for recent developments) that measures in $\operatorname{MAH}(X, \omega)$ have the following strong integrability property: if $\mu \in \operatorname{MAH}(X, \omega)$, then

$$
\exp (-\varepsilon \operatorname{PSH}(X, \omega)) \subset L^{1}(\mu) \text {, for some } \varepsilon>0 \text {. }
$$


This is a useful generalization of Skoda's celebrated integrability theorem (see [Sk, Ze]).

It is natural to wonder whether condition $(\dagger)$ characterizes $\operatorname{MAH}(X, \omega)$. This is the case when $n=1$ (see [DS] and Subsection 4.1). In this note we show that such a characterization still holds in higher dimensions provided the measures under consideration have symmetries:

Theorem C. Let $\mu$ be a probability measure with finitely many isolated singularities of radial or toric type. Then $\mu$ belongs to $\operatorname{MAH}(X, \omega)$ if and only if $(\dagger)$ is satisfied.

Next we turn our attention to the general big cohomology class setting. To this end we choose a smooth $(1,1)$-form $\theta$ representing $\Theta$. Observe that in general one cannot have $\theta \geq 0$. Analogously to the Kähler setting we can nevertheless define $\operatorname{PSH}(X, \theta)$ as the set of functions which are defined again as being locally equal to sum of a plurisubharmonic and a smooth function and any such function $\varphi$ should satisfy $\theta+d d^{c} \varphi \geq 0$. Observe that by assumption such functions exist, although they all may be singular.

It follows from the regularization theorem of the first author [D4] that we can find a strictly positive closed $(1,1)$ current $T_{+}=\theta+d d^{c} \varphi_{+}$which represents $\Theta$ and has analytic singularities, that is there exists $c>0$ such that locally on $X$ we have

$$
\varphi_{+}=c \log \sum_{j=1}^{N}\left|f_{j}\right|^{2} \bmod C^{\infty}
$$

where $f_{1}, \ldots, f_{N}$ are local holomorphic functions. Such a current $T_{+}$is then smooth on a Zariski open subset $\Omega$, and the ample locus $\operatorname{Amp}(\Theta)$ of $\Theta$ is defined as the largest such Zariski open subset (which exists by the Noetherian property of closed analytic subsets). Therefore any $\theta$-psh function $\psi$ with minimal singularities is locally bounded on the ample locus. Here having minimal singularity means that given any other $\theta$-psh function $\varphi$ one has the inequality

$$
\varphi \leq \psi+O(1) .
$$

According to [BEGZ] we can then define the (non-pluripolar) product $\left\langle\left(\theta+d d^{c} \varphi\right)^{n}\right\rangle$, and in case $\varphi$ has minimal singularities, the total mass of this measure is independent of $\varphi$ and equals

$$
\int_{X}\left\langle\left(\theta+d d^{c} \varphi\right)^{n}\right\rangle=: \operatorname{Vol}(\Theta)>0 .
$$

It is therefore meaningful to study the (normalized) Monge-Ampère equation

$$
M A(\varphi):=\frac{1}{\operatorname{Vol}(\Theta)}\left(\theta+d d^{c} \varphi\right)^{n}=\mu,
$$

for a given probability measure $\mu$ vanishing on pluripolar sets.

When $\mu=f d V$ is absolutely continuous with respect to Lebesgue measure with density $f \in L^{p}(X), p>1$, there is a unique solution modulo additive constant which turns out to have minimal singularities [BEGZ]. The solution is known to be globally continuous on $X$ when the cohomology class $\Theta$ is moreover semi-positive ([EGZ3]).

In this context we prove the following analogue of Theorem A:

Theorem D. Let $\mu$ be a probability measure absolutely continuous with respect to a fixed smooth volume form, with density $f \in L^{p}(X), p>1$. Let $\varphi$ be a weak solution of the Monge-Ampère equation $M A(\varphi)=\mu$. Then for any $0<\alpha<2 /(1+n q), \varphi$ is Hölder-continuous of exponent $\alpha$ locally in the ample locus Amp $(\Theta)$ of $\Theta$ (here $q$ denotes the conjugate exponent of $p$ ).

The note is organized as follows. In Section 2 we recall all the basic facts and introduce the necessary definitions. Theorems A and $A^{*}$ are proved in Section 3. After recalling the one dimensional theory in Subsection 4.1, we establish the characterization of $\operatorname{MAH}(X, \omega)$ in Subsection 4.2. This allows us to prove Theorem B (in Subsection 4.3) and derive further interesting consequences. The case of measures with symmetries is handled in Section 5. Theorem $D$ is proved in Section 6. In the Appendix we briefly explain how bounds on the curvature 
allow to control the differential of the exponential mapping, a technical information needed in the proof of Theorem $A^{*}$.

\section{PRELIMINARIES}

2.1. Curvature and regularization. Let $X$ be a compact Kähler manifold equipped with a fundamental Kähler form $\omega$ given in local coordinates by

$$
\omega=\frac{i}{2} \sum_{k, j} g_{k \bar{j}} d z^{k} \wedge d \bar{z}^{j}
$$

Its bisectional curvature tensor in those local coordinates is defined by

$$
R_{i \bar{j} k \bar{l}}:=-\frac{\partial^{2} g_{k \bar{l}}}{\partial z_{i} \partial \bar{z}_{j}}+\sum_{p, q=1}^{n} g^{p \bar{q}} \frac{\partial g_{p \bar{l}}}{\partial \bar{z}_{j}} \frac{\partial g_{k \bar{q}}}{\partial z_{i}}
$$

with $g^{p \bar{q}}$ denoting the inverse transposed matrix of $g_{p \bar{q}}$ i.e., $\sum_{q=1}^{n} g^{p \bar{q}} g_{s \bar{q}}=\delta_{p s}$. It is a classical fact that in the Kähler case the bisectional curvature tensor coincides with the Levi-Civita curvature tensor. We say that the bisectional curvature is bounded by $A>0$ if for any $z \in X$ and any vectors $Z, Y \in T_{z} X, Z, Y \neq 0$ we have the inequality

$$
\left|\sum_{i, j, k, l}^{n} R_{i \bar{j} k \bar{l}}(z) Z_{i} \bar{Z}_{j} Y_{k} \bar{Y}_{l}\right| \leq A\|Z\|_{\omega}^{2}\|Y\|_{\omega}^{2} .
$$

Analogously the bisectional curvature is bounded from below (resp. from above) by $A$ if

$$
\sum_{i, j, k, l}^{n} R_{i \bar{j} k \bar{l}}(z) Z_{i} \bar{Z}_{j} Y_{k} \bar{Y}_{l} \geq A\|Z\|_{\omega}^{2}\|Y\|_{\omega}^{2}, \quad(\text { resp. } \leq)
$$

respectively. It is easy to check that the existence of such bounds is independent of the choice of local coordinates.

Recall that if $u$ is a psh function in a domain in $\mathbb{C}^{n}$ then a convolution with a radial smoothing kernel preserves positivity of $d d^{c} u$. For non-flat metrics, this may not be the case any longer. However, an approximation technique due to the first author allows to control "the negative part" of the smooth form. It is described in detail in [D1] and [D4]. Here we shall briefly highlight its main features.

Consider the exponential mapping from the tangent space of a given point $z \in X$

$$
\exp _{z}: T_{z} X \ni \zeta \mapsto \exp _{z}(\zeta) \in X
$$

which is defined by $\exp _{z}(\zeta)=\gamma(1)$ with $\gamma$ being the geodesic starting from $z$ with initial velocity $\gamma^{\prime}(0)=\zeta$. Given any function $u \in L^{1}(X)$, we define its $\delta$-regularization $\rho_{\delta} u$ to be

$$
\rho_{\delta} u(z)=\frac{1}{\delta^{2 n}} \int_{\zeta \in T_{z} X} u\left(\exp _{z}(\zeta)\right) \rho\left(\frac{|\zeta|_{\omega}^{2}}{\delta^{2}}\right) d V_{\omega}(\zeta), \delta>0
$$

according to [D1]. Here $\rho$ is a smoothing kernel, $|\zeta|_{\omega}^{2}$ stands for $\sum_{i, j=1}^{n} g_{i \bar{j}}(z) \zeta_{i} \bar{\zeta}_{j}$, and $d V_{\omega}(\zeta)$ is the induced measure $\frac{1}{2^{n} n !}\left(d d^{c}|\zeta|_{\omega}^{2}\right)^{n}$. This may be formally extended as a function on $X \times \mathbb{C}$ by putting $U(z, w):=\rho_{\delta} u(z)$ for $w \in \mathbb{C},|w|=\delta$. The introduction of the variable $w$ is convenient for an application of Kiselman minimum principle [Ki1, Ki2] to that function. It should be noticed that in [D4] the riemannian exponential map "exp" has been replaced by a "holomorphic counterpart" exph, which is defined as the holomorphic part of the Taylor expansion of $\zeta \mapsto \exp _{z}(\zeta)$ (the reason is that the calculations then become somewhat simpler, especially in the non Kähler case, but this is not technically necessary; thanks to a well known theorem of E. Borel, such a formal expansion can always be achieved by a smooth function exph : $T X \rightarrow X)$. The function exph is however not uniquely defined, and this weakens the intrinsic character of the estimates. Therefore, we stick here to the more usual riemannian exp function. 
The estimates obtained in [D1] show that all results of [D4] and [BD] are still valid with the unmodified definition of $\rho_{\delta} u$, at least when $(X, \omega)$ is Kähler. By Lemma 8.2 of [D1], the exponential function

$$
\exp : T X \rightarrow X, \quad T X \ni(z, \zeta) \mapsto \exp _{z}(\zeta) \in X, \zeta \in T_{z} X
$$

satisfies the following properties:

(1) $\exp$ is a $\mathcal{C}^{\infty}$ smooth mapping;

(2) $\forall z \in X, \exp _{z}(0)=z$ and $d_{\zeta} \exp (0)=\operatorname{Id}_{T_{z} X}$;

(3) $\forall z \in X$ the map $\zeta \rightarrow \exp _{z} \zeta$ has a third order Taylor expansion at $\zeta=0$ of the form

$$
\left|\exp _{z}(\zeta)_{m}-z_{m}-\zeta_{m}-\frac{1}{2} \sum_{j, k, l} R_{j \bar{k} l \bar{m}}\left(\bar{z}_{k}+\frac{1}{3} \bar{\zeta}_{k}\right) \zeta_{j} \zeta_{l}\right| \leq C\left(|\zeta|^{2}(|z|+|\zeta|)^{2}\right), \quad|\zeta|<r,
$$

for small enough $r>0$. The expansion is valid in holomorphic normal coordinates with respect to the Kähler metric.

It is convenient to select a particular smoothing kernel, namely $\rho: \mathbb{R}_{+} \rightarrow \mathbb{R}_{+}$by setting

$$
\rho(t)= \begin{cases}\frac{\eta}{(1-t)^{2}} \exp \left(\frac{1}{t-1}\right) & \text { if } 0 \leq t \leq 1 \\ 0 & \text { if } t>1\end{cases}
$$

with a suitable constant $\eta$, such that

$$
\int_{\mathbb{C}^{n}} \rho\left(\|z\|^{2}\right) d V(z)=1
$$

$\left(d V\right.$ denotes the Lebesgue measure in $\left.\mathbb{C}^{n}\right)$.

The crucial estimate of the Hessian of $U(z, w)$ given in [D4], Proposition 3.8 (see also [D1], Proposition 8.5), coupled with Kiselman's theorem provide a lemma stated in this form in [BD, Lemma 1.12]:

Lemma 2.1. Fix any bounded $\omega$-psh function $u$ on a compact Kähler manifold $(X, \omega)$. Let $U(z, w)$ be its regularization as defined above. Define the Kiselman-Legendre transform with level c by

$$
u_{c, \delta}:=\inf _{0 \leq t \leq \delta}\left[U(z, t)+K t^{2}-K \delta^{2}-c \log \left(\frac{t}{\delta}\right)\right] .
$$

Then for some positive constant $K$ depending on the curvature, the function $U(z, t)+K t^{2}$ is increasing in $t$ and one has the following estimate for the complex Hessian:

$$
\omega+d d^{c} u_{c, \delta} \geq-\left(A \min \{c, \lambda(z, \delta)\}+K \delta^{2}\right) \omega,
$$

where $A$ is a lower bound of the negative part of the bisectional curvature of $\omega$, while

$$
\lambda(z, t):=\frac{\partial}{\partial \log t}\left(U(z, t)+K t^{2}\right) .
$$

2.2. Jensen formula and uniformly bounded geometry. The classical Jensen formula (see, for example [BT1]) for a $\mathcal{C}^{2}$ function $u$ defined in a ball $B(z, 2 \delta)$ in $\mathbb{C}^{n}$ says that

$$
\left(\check{u}_{\delta}-u\right)(z)=\frac{2 n}{\delta^{2 n} \sigma_{2 n-1}} \int_{0}^{\delta} r^{2 n-1} \int_{0}^{r} t^{1-2 n} \int_{|\zeta| \leq t} \Delta u(z+\zeta) d V(\zeta) d t d r,
$$

where $\check{u}_{\delta}$ is the average of $u$ over $B(z, \delta)$ and $\sigma_{2 n-1}$ denotes the total surface measure of the unit sphere. Now, if $u$ is defined in a large set, then the integration of the above formula in $z$ provides an estimate of the integral of $\delta^{-2}\left(\check{u}_{\delta}-u\right)$ in terms of the integral of the Laplacian of $u$. We need such an estimate on compact Kähler manifolds which is uniform as long as the geometry of manifolds is bounded in a certain sense. 
Definition 2.2. Consider a family $\left(X_{s}, \omega_{s}\right)$ of compact Kähler manifolds. We shall say that it has uniformly bounded geometry if

1) the diameter $\operatorname{diam}\left(X_{s}, \omega_{s}\right)$ is uniformly bounded,

2) their bisectional curvatures are uniformly bounded,

3) the injectivity radius is uniformly bounded from below.

By well-known estimates $[\mathrm{HK}]$, it then follows that the total volumes $\operatorname{Vol}_{\omega_{s}}\left(X_{s}\right):=\int_{X_{s}} \omega_{s}^{n}$ are uniformly bounded above and below by constants $C$ and $C^{-1}$ independent of $s$.

It turns out that such bounds are enough to ensure various interesting geometric and analytic bounds. Note in particular that they imply lower bounds on the Tian $\alpha$ invariants for the classes of $\omega_{s}$-psh functions which does not depend on $s$ (see [BEGZ]). In potential applications $X$ will usually stay fixed, while the Kähler forms may vary. Note that all conditions are obviously satisfied if the forms $\omega_{s}$ are bounded in $\mathcal{C}^{\infty}$ topology and uniformly positive; this can be achieved by selecting appropriate representatives when the cohomology classes $\left[\omega_{s}\right]$ are given and contained in a fixed relatively compact region of the Kähler cone of $X$. Thus an interesting case to treat would be when the classes $\left[\omega_{s}\right]$ approach the boundary of this cone. Unfortunately this may in general lead to a blow-up of the curvature and for this reason our argument cannot be applied to study the limiting behavior. On the other hand the method works if the forms $\omega_{s}$ approximate a $\mathcal{C}^{1,1}$ form $\omega$ in a fixed cohomology class provided that the curvatures of $\omega_{s}$ stay bounded.

We can now state a lemma to be used in the next section.

Lemma 2.3. Assume $\left(X_{s}, \omega_{s}\right)$ is a family of compact Kähler manifolds with uniformly bounded geometry. Let $u_{s}$ be continuous $\omega_{s}$-psh functions normalized by $\min _{X_{s}} u_{s}=1, \max _{X_{s}} u_{s} \leq B$ for some fixed constant $B$. If $\rho_{\delta} u_{s}$ is the regularization of $u_{s}$ defined as in (2.1) then for $\delta$ small enough we have

$$
\int_{X_{s}} \frac{\rho_{\delta} u_{s}-u_{s}}{\delta^{2}} \omega_{s}^{n} \leq C_{0}
$$

where $C_{0}$ only depends on $B$ and the constants involved in the uniform bounds on the geometry.

Proof. Let us fix $s$ and omit it in the notation for simplicity. By definition

$$
\begin{aligned}
\rho_{\delta} u(z) & =\int_{\zeta \in T_{z} X} u\left(\exp _{z} \zeta\right) \rho\left(\frac{|\zeta|_{\omega}^{2}}{\delta^{2}}\right) \frac{d V_{\omega}(\zeta)}{\delta^{2 n}}=\int_{x \in X} u(x) \rho\left(\frac{\left|\log _{z} x\right|_{\omega}^{2}}{\delta^{2}}\right) \frac{d V_{\omega}\left(\log _{z} x\right)}{\delta^{2 n}} \\
& =\int_{x \in X} u(x) K_{\delta}(z, x)
\end{aligned}
$$

where $x \mapsto \zeta=\log _{z} x$ is the inverse of $\zeta \mapsto x=\exp _{z}(\zeta)$. The map $(z, x) \mapsto\left(z, \log _{z} x\right)$ defines a diffeomorphism from a neighborhood of the diagonal in $X \times X$ onto a neighborhood of the zero section of $T X$ by the implicit function theorem. Here

$$
K_{\delta}(z, x)=\frac{1}{\delta^{2 n}} \rho\left(\frac{\left|\log _{z} x\right|_{\omega}^{2}}{\delta^{2}}\right) d V_{\omega}\left(\log _{z} x\right)
$$

is the semipositive $(n, n)$ form on $X \times X$ defined as the pull-back of $\rho\left(|\zeta|_{\omega}^{2} / \delta^{2}\right) d V_{\omega}(\zeta) / \delta^{2 n}$ by $(z, x) \mapsto \zeta=\log _{z} x$; it can be viewed as a kernel with compact support in a neighborhood of the diagonal of $X \times X$. By definition, we have $\int_{x \in X} K_{\delta}(z, x)=1$ (as is clear by taking $u \equiv 1$ ), thus

$$
u(z)=\int_{x \in X} u(z) K_{\delta}(z, x)
$$


Therefore

$$
\begin{aligned}
\int_{X}\left(\rho_{\delta} u(z)-u(z)\right) d V_{\omega}(z) & =\int_{(x, z) \in X \times X}(u(x)-u(z)) K_{\delta}(z, x) \wedge d V_{\omega}(z) \\
& =\int_{(x, z) \in X \times X} u(x)\left(K_{\delta}(z, x) \wedge d V_{\omega}(z)-K_{\delta}(x, z) \wedge d V_{\omega}(x)\right)
\end{aligned}
$$

thanks to a change of variable $(z, x) \mapsto(x, z)$. In order to finish the proof we need the following lemma which establishes a pointwise bound for the kernel:

Lemma 2.4. If $d_{\omega}(z, x) \leq \delta$, then

$$
\left|\left(K_{\delta}(z, x) \wedge d V_{\omega}(z)-K_{\delta}(x, z) \wedge d V_{\omega}(x)\right)\right| \leq C \delta^{2-2 n} d V_{\omega}(z) \wedge d V_{\omega}(x),
$$

for some uniform constant $C$ which only depends on the curvature of $\omega$. If $d_{\omega}(z, x)>\delta$, then $K_{\delta}(z, x) \wedge d V_{\omega}(z)=K_{\delta}(x, z) \wedge d V_{\omega}(x)=0$.

Proof. Given the symmetry of $\left|\log _{z}(x)\right|_{\omega}=\left|\log _{x}(z)\right|_{\omega}=d_{\omega}(z, x)$, it is enough to bound the $(2 n, 2 n)$-form $d V_{\omega}\left(\log _{z} x\right) \wedge d V_{\omega}(z)-d V_{\omega}\left(\log _{x} z\right) \wedge d V_{\omega}(x)$. The last assertion follows from the fact that $\rho\left(\frac{\left|\log _{z} x\right|_{\omega}^{2}}{\delta^{2}}\right)=\rho\left(\frac{\left|\log _{x} z\right|_{\omega}^{2}}{\delta^{2}}\right)=0$ if $d_{\omega}(z, x)>\delta$.

We now establish the first part of the lemma. Set $\zeta=\log _{z} x$ (i.e. $x=\exp _{z}(\zeta)$ ) and $y=$ $\exp _{z}\left(\frac{\zeta}{2}\right)=\exp _{z}\left(\frac{1}{2} \log _{z}(x)\right)$ (the mid-point of the geodesic joining $z$ and $x$ ). Observe that from the expansion (2.2) applied at $y$ (which is identified with zero in this system of normal coordinates) we have

$$
\zeta_{m}=\log _{z}(x)_{m}=x_{m}-z_{m}-\frac{1}{2} \sum_{j, k, l} R_{j \bar{k} l \bar{m}}\left(\bar{z}_{k}+\frac{1}{3}\left(\bar{x}_{k}-\bar{z}_{k}\right)\right)\left(x_{j}-z_{j}\right)\left(x_{l}-z_{l}\right)+O\left(\|z-x\|^{4}\right) .
$$

Now (2.7) yields

$$
d \zeta_{m}=d\left(\log _{z} x\right)_{m}=d x_{m}-d z_{m}+O\left(\|z-x\|^{2}\right)(d x, d z)
$$

with an $O(\ldots)$ term depending only on the curvature. By the choice of the center $y$ we have $z_{j}=\frac{1}{2}\left(z_{j}-x_{j}\right)+O\left(\|z-x\|^{2}\right)$, where the $O(\ldots)$ term again only depends on the curvature. Thus the expansion

$$
d V_{\omega}(\zeta)=\frac{\omega(z)^{n}}{n !}(\zeta)=\left(1-\sum_{j, k, l} R_{j \bar{k} l} \bar{z}_{j} \bar{z}_{k}+O\left(\|z\|^{3}\right)\right) \frac{i}{2} d \zeta_{1} \wedge d \bar{\zeta}_{1} \wedge \ldots \wedge \frac{i}{2} d \zeta_{n} \wedge d \bar{\zeta}_{n}
$$

at any given point $z$ yields

$$
d V_{\omega}\left(\log _{z} x\right)=\bigwedge_{j=1}^{n} \frac{i}{2}\left(d x_{j}-d z_{j}\right) \wedge\left(d \bar{x}_{j}-d \bar{z}_{j}\right)+O\left(\|z-x\|^{2}\right)
$$

Thus, by taking the product with $d V_{\omega}(z)$, exchanging $x$ and $z$, and then subtracting and dividing by $\delta^{2 n}$, we obtain the desired bound

$$
\frac{d V_{\omega}\left(\log _{z} x\right) \wedge d V_{\omega}(z)-d V_{\omega}\left(\log _{x} z\right) \wedge d V_{\omega}(x)}{\delta^{2 n}}=\frac{O\left(\|z-x\|^{2}\right)}{\delta^{2 n}} d V_{\omega}(z) \wedge d V_{\omega}(x) .
$$

The appendix implies that $O(\ldots)$ depends only on global bounds for the geometry. 
We can now use Fubini's theorem and the estimates on the kernel to obtain

$$
\begin{array}{rl}
\int_{(x, z) \in X \times X} & u(x)\left(K_{\delta}(z, x) \wedge d V_{\omega}(z)-K_{\delta}(x, z) \wedge d V_{\omega}(x)\right) \\
& =\int_{x \in X} \int_{z \in B(x, \delta)} u(x)\left(K_{\delta}(z, x) \wedge d V_{\omega}(z)-K_{\delta}(x, z) \wedge d V_{\omega}(x)\right) \\
\leq & \int_{x \in X} \int_{z \in B(x, \delta)}|u(x)| C \delta^{2-2 n} d V_{\omega}(z) \wedge d V_{\omega}(x) \\
\leq & \int_{x \in X} B C \delta^{2} d V_{\omega}(x) \leq C_{0} \delta^{2}
\end{array}
$$

as claimed.

2.3. The $\mathcal{H}(\alpha)$ condition and measures uniformly dominated by capacity. A fundamental tool in the study of $\omega$-psh functions is the relative capacity modelled on the BedfordTaylor relative capacity $([\mathrm{BT} 2])$.

Definition 2.5. Let $(X, \omega)$ be a compact Kähler manifold. Given a Borel subset $K$ of $X$, we define its relative capacity with respect to $\omega$ by

$$
\operatorname{Cap}_{\omega}(K):=\sup \left\{\int_{K}\left(\omega+d d^{c} \rho\right)^{n} \mid \rho \in \operatorname{PSH}(X, \omega), 0 \leqslant \rho \leqslant 1\right\} .
$$

The following classes have been considered in [EGZ1]:

Definition 2.6. Let $\mu$ be a probability measure on a compact Kähler manifold $(X, \omega)$. We say that $\mu$ belongs to the class $\mathcal{H}(\alpha), \alpha>0$ (alternatively, that $\mu$ statisfies the $\mathcal{H}(\alpha)$ property), if there exists $C_{\alpha}>0$ such that for any compact $K \subset X$,

$$
\mu(K) \leq C_{\alpha} \operatorname{Cap}_{\omega}(K)^{1+\alpha}
$$

If this holds for any $\alpha>0$, we say that $\mu$ satisfies $\mathcal{H}(\infty)$.

It was proved in $[\mathrm{K} 1, \mathrm{~K} 3]$ that measures of the type $\mu=f \omega^{n}$ with a density $f$ in $L^{p}$ for some $p>1$ do satisfy $\mathcal{H}(\infty)$ (see also [Ze]). A slightly stronger notion was introduced in [DZ]:

Definition 2.7. We say that a probability measure $\mu$ is dominated by capacity for $L^{p}$ functions if there exists constants $\alpha>0$ and $\beta>0$, such that for any compact $K \subset X$ and non-negative $f \in L^{p}(\mu)$ with $p>1$, one has for some constant $C$ independent of $K$ that

$$
\mu(K) \leqslant C \cdot \operatorname{Cap}_{\omega}(K)^{1+\alpha} \text { and } \int_{K} f \mu \leqslant C \cdot \operatorname{Cap}_{\omega}(K)^{1+\beta} .
$$

Both notions are variations on the condition (A) introduced by fith named author in [K1]. These conditions, which are actually stronger than condition (A), ensure the existence of bounded solutions $u$ to

as long as $\int_{X} f d \mu=1$.

$$
M A(u)=f \mu,
$$

Note that the condition $\mathcal{H}(\infty)$ is equivalent to domination by capacity for $L^{\infty}$ functions by a simple application of the Hölder inequality.

2.4. Big cohomology classes. Let $X$ be a compact Kähler manifold of dimension $n$, and $\Theta=\{\theta\} \in H^{1,1}(X, \mathbb{C}) \cap H^{2}(X, \mathbb{R})$ a big cohomology class with a smooth representative $\theta$.

We introduce the extremal function $V_{\theta}$ defined by

$$
V_{\theta}(x):=\sup \left\{\varphi(x) \mid \varphi \in P S H(X, \theta), \sup _{X} \varphi \leq 0\right\},
$$

where $P S H(X, \theta)$ is the set of all $\theta$-plurisubharmonic functions on $X$. The function $V_{\theta}$ is a $\theta$-psh function with minimal singularities. 
Similarly to the Kähler case we define the relative capacity:

Definition 2.8. Let $X$ be a compact Kähler manifold. Given a Borel subset $K$ of $X$, we define its relative capacity with respect to $\theta$ by

$$
\operatorname{Cap}_{\theta}(K):=\sup \left\{\int_{K}\left(\theta+d d^{c} \rho\right)^{n} \mid \rho \in \operatorname{PSH}(X, \theta), V_{\theta}(x)-1 \leqslant \rho \leqslant V_{\theta}(x)\right\} .
$$

Observe that contrary to the Kähler case competitors to maximize the right hand side have minimal singularities but are in general unbounded. The Monge-Ampère measures in the definition are only considered outside the polar locus $\left\{x \in X \mid V_{\theta}(x)=-\infty\right\}$. Observe that the latter depends on the cohomology class $\{\theta\}$ but not on the choice of its representative $\theta$.

Most definitions from the Kähler setting have their big counterparts, we refer the readers to [BEGZ] for details and more background regarding big cohomology classes. In particular we can apply the same convolution procedure to any $\theta$-psh function, as well as the Kiselman-Legendre transform.

In order to prove Theorem D we shall need a stability estimate proved in [GZ2]:

Proposition 2.9. Assume that $\mu$ is a probability measure absolutely continous with respect to a smooth volume form $d V, d \mu=f d V$, where $f \in L^{p}(X)$ with $p>1$. Let $\varphi, \psi$ be $\theta$ plurisubharmonic functions such that $M A(\varphi)=\mu,-M_{0}+V_{\theta} \leq \varphi \leq V_{\theta}$ and $\psi \leq V_{\theta}$ on $X$, for some positive constant $M_{0}>0$. Then for any exponent $0<\gamma<\frac{1}{n q+1}$, there exists a constant $B_{0}=B_{0}\left(p, \gamma, M_{0}\right)>0$ such that

$$
\sup _{X}(\psi-\varphi)_{+} \leq B_{0}\left\|(\psi-\varphi)_{+}\right\|_{L^{1}(X)}^{\gamma} .
$$

\section{Proof of Theorems A And A*}

Proof of Theorem A. Fix $u \in P S H(X, \omega)$ such that $M A(u)=\mu$. Denote by $A-1=A^{\prime}>0$ a bound for the curvature of $(X, \omega)$. By [K1] $u$ is continuous, so assume that $\min _{X} u=1$ and denote by $B:=\max _{X} u$ the maximum of $u$. Consider $\rho_{\delta} u$ - the regularization of the $\omega$-psh function $u$ defined in (2.1).

Let us set for $\delta>0$ and $\alpha>0$,

$$
E(\delta, \alpha):=\left\{\left(\rho_{\delta} u-u\right)(z)>\delta^{\alpha}\right\}
$$

Let $0<\alpha_{1}<\frac{2}{q n+1}$. Choose $\varepsilon>0, \alpha, \alpha_{0}$ such that

$$
\alpha_{1}<\alpha<\alpha_{0}<2-\alpha_{0} q(n+\varepsilon) .
$$

Set $\theta:=e^{-3 A B}$. Recall (Lemma 2.1) that there exists a constant $K$ which only depends on the curvature such that the functions $\rho_{\delta} u+K \delta^{2}$ are increasing in $\delta$. Note that for $\delta$ small enough $\theta^{\alpha_{1}} \delta^{\alpha_{1}} \geq \delta^{\alpha_{0}}+K \delta^{2}\left(1-\theta^{2}\right)$. Altogether this implies that $E\left(\delta, \alpha_{0}\right) \supset E\left(\theta \delta, \alpha_{1}\right)$.

We want to show that $E\left(\theta \delta, \alpha_{1}\right)$ is empty. Recall the definition of the Kiselman-Legendre transform at level $\delta^{\alpha}$ (see Lemma 2.1)

$$
U_{\delta}=\inf _{t \in[0, \delta]}\left(\rho_{t} u+K t^{2}-\delta^{\alpha} \log \frac{t}{\delta}-K \delta^{2}\right),
$$

where $K$ is chosen as in the formula (2.4). It follows from [D1] that the same $K$ can be chosen for a family of manifolds with uniformly bounded geometry. In what follows $\delta_{0}$ and $\delta_{j}, c_{j}, j=1,2,3$; denote constants which are uniform if the geometry is uniformly bounded and $\|f\|_{p}$ stays bounded.

By Lemma 2.1

$$
\omega+d d^{c} U_{\delta} \geq-\left[(A-1) \delta^{\alpha}+K \delta^{2}\right] \omega>-A \delta^{\alpha} \omega+2 \delta^{\alpha_{0}} \omega
$$


for $0<\delta<\delta_{0}$, where $\delta_{0}>0$ is small enough. Therefore

$$
u_{\delta}:=\frac{1}{1+A \delta^{\alpha}} U_{\delta}
$$

is $\omega$-psh on $X$ and satisfies

$$
\omega+d d^{c} u_{\delta} \geq \delta^{\alpha_{0}} \omega
$$

provided $A \delta^{\alpha}<1$, which we can safely assume. From Lemma 2.3 we have

$$
\int_{X}\left|\rho_{\delta} u-u\right| \omega^{n} \leq c_{1} \delta^{2}
$$

for $0<\delta<\delta_{0}$. Therefore for $E_{0}=E\left(\delta, \alpha_{0}\right)=\left\{\left(\rho_{\delta} u-u\right)(z)>\delta^{\alpha_{0}}\right\}$ we have

$$
\int_{E_{0}} \omega^{n} \leq c_{1} \delta^{2-\alpha_{0}}
$$

and, by Hölder inequality,

$$
\int_{E_{0}} f \omega^{n} \leq c_{2} \delta^{\left(2-\alpha_{0}\right) / q}
$$

Let us modify $f$ setting $g=0$ on $E_{0}$ and $g=c f$ elsewhere, with $c$ such that total integrals of $f$ and $g$ are equal. Solve for continuous $\omega$-psh function $v$ (comp. [K2])

$$
\left(\omega+d d^{c} v\right)^{n}=g \omega^{n}, \quad \max (u-v)=\max (v-u) .
$$

Observe that $\|f-g\|_{L^{1}(X)}=2 \int_{E_{0}} f \omega^{n} \leq 2 c_{2} \delta^{\left(2-\alpha_{0}\right) / q}$. Then by [DZ] there exists $c_{3}\left(c_{3}\right.$ depends additionally on $\varepsilon>0$ ) such that

$$
\|u-v\|_{L^{\infty}} \leq c_{3} \delta^{\frac{2-\alpha_{0}}{q(n+\varepsilon)}} .
$$

We claim that there exist small enough constants $\delta_{1}>\delta_{2}>\delta_{3}>0$ such that for any $0<\delta<\delta_{3}$ there is a set inclusion

$$
E\left(\theta \delta, \alpha_{1}\right) \subset\left\{u_{\delta}-v>\delta^{\alpha}\right\} \subset E\left(\delta, \alpha_{0}\right) .
$$

Indeed, take $z$ in $E\left(\theta \delta, \alpha_{1}\right)$. By Lemma 2.1, the function $\rho_{t} u+K t^{2}$ is increasing in $t \in[0, \delta]$. Thus for $t \in[\theta \delta, \delta]$,

$$
\rho_{t} u(z)-u(z)=\rho_{t} u(z)-\rho_{\theta \delta} u+\rho_{\theta \delta} u-u(z) \geq K(\theta \delta)^{2}-K t^{2}+(\theta \delta)^{\alpha_{1}} \geq(\theta \delta)^{\alpha_{1}}-K \delta^{2},
$$

and for $t<\theta \delta$, since $\theta=e^{-3 A B}$, we have

$$
-\delta^{\alpha} \log (t / \delta) \geq 3 A B \delta^{\alpha} .
$$

Therefore

$$
\left(U_{\delta}-u\right)(z) \geq \min \left(\left(e^{-3 A B} \delta\right)^{\alpha_{1}}-K \delta^{2}, 3 A B \delta^{\alpha}\right)=3 A B \delta^{\alpha}
$$

for $0<\delta<\delta_{1}$, where $\delta_{1}>0$ is small enough (we can safely assume that $\delta_{1}<\delta_{0}$ ). Hence, by $(3.3)$

$$
\left(U_{\delta}-v\right)(z) \geq 3 A B \delta^{\alpha}-c_{3} \delta^{\frac{2-\alpha_{0}}{q(n+\varepsilon)}}>2 A B \delta^{\alpha},
$$

for $\delta<\delta_{2}$, where $0<\delta_{2}<\delta_{1}$ is small enough. Observe that

$$
U_{\delta}-u_{\delta} \leq A B \delta^{\alpha} .
$$

Since $A B \geq 1$, it follows that $u_{\delta}(z)-v(z)>A B \delta^{\alpha}>\delta^{\alpha}$ for $\delta<\delta_{2}$, which proves the first inclusion $E\left(\theta \delta, \alpha_{1}\right) \subset\left\{u_{\delta}-v>\delta^{\alpha}\right\}$ in $(3.4)$.

To prove the second inclusion, take $z \notin E\left(\delta, \alpha_{0}\right)$. Since, under our assumptions

$$
u_{\delta}<U_{\delta} \leq \rho_{\delta} u
$$

we get, applying (3.3)

$$
\left(u_{\delta}-v\right)(z) \leq\left(\rho_{\delta} u-u\right)(z)+c_{3} \delta^{\frac{2-\alpha_{0}}{q(n+\varepsilon)}} \leq \delta^{\alpha_{0}}+c_{3} \delta^{\frac{2-\alpha_{0}}{q(n+\varepsilon)}}<\delta^{\alpha},
$$


for $0<\delta<\delta_{3}$, where $0<\delta_{3}<\delta_{2}$ is small enough. This proves our second inclusion

$$
\left\{u_{\delta}-v>\delta^{\alpha}\right\} \subset E\left(\delta, \alpha_{0}\right)
$$

for $0<\delta<\delta_{3}$ and completes the proof of (3.4).

Now we want to apply the comparison principle do deduce from (3.4) that the set $E\left(\theta \delta, \alpha_{1}\right)$ is empty for $\delta>0$ small enough. Let us fix $0<\delta<\delta_{3}$ and recall that $E_{0}=E\left(\delta, \alpha_{0}\right)$. From (3.4) and the comparison principle [K2], if follows that

$$
\int_{\left\{u_{\delta}>v+\delta^{\alpha}\right\}}\left(d d^{c} u_{\delta}+\omega\right)^{n} \leq \int_{\left\{u_{\delta}>v+\delta^{\alpha}\right\}}\left(d d^{c} v+\omega\right)^{n} \leq \int_{E_{0}}\left(d d^{c} v+\omega\right)^{n}=\int_{E_{0}} g \omega^{n}=0 .
$$

Since $u_{\delta}$ is $\omega$-psh and $\left(\omega+d d^{c} u_{\delta}\right)^{n} \geq \delta^{n \alpha_{0}} \omega^{n}$, it follows that the volume of the set $\left\{u_{\delta}>v+\delta^{\alpha}\right\}$ is zero. Hence it is empty, since $u_{\delta}$ and $v$ are $\omega$-psh functions. Therefore from (3.4), it follows that the set $E\left(\theta \delta, \alpha_{1}\right)$ is also empty. Setting $\eta=\theta \delta=\delta e^{-3 A B}$, we obtain

$$
\rho_{\eta} u-u \leq e^{3 \alpha_{1} A B} \eta^{\alpha_{1}}
$$

for $0<\eta<\eta_{0}=e^{-3 \alpha A B} \delta_{3}$.

Note that the above inequality means that locally ["words permutation"] the $\eta$ - convolution of $u$ is no more than $u$ plus some constant of order $\eta^{\alpha_{1}}$. Thus repeating the local argument from [GKZ] one obtains that the supremum of $u$ in a coordinate ball of radius $\eta$ and center $z$ is also controlled by $u(z)$ and a constant of order $\eta^{\alpha_{1}}$. This proves that $u$ is Hölder continuous with exponent $\alpha_{1}$.

Note that in the proof above we could choose the same $\delta_{1}, \delta_{2}$ and $\delta_{3}$ for uniform $\alpha_{j}, c_{j}$. Thus, following the lines of this proof, one can obtain an analogous result for families of manifolds with uniformly bounded geometry.

Theorem 3.1 (Theorem $\left.\mathrm{A}^{*}\right)$. Let $\left(X_{s}, \omega_{s}\right)$ be a family of $n$-dimensional compact Kähler manifolds with uniformly bounded geometry. Consider the Monge-Ampère equations

$$
\left(\omega_{s}+d d^{c} u_{s}\right)^{n}=f_{s} \omega_{s}^{n}, \quad \sup _{X_{s}} u_{s}=0,
$$

where $\int_{X_{s}} f_{s} \omega_{s}^{n}=\int_{X_{s}} \omega_{s}^{n}$.

If $\|f\|_{L^{p}\left(\omega_{s}^{n}\right)} \leq C$ are uniformly bounded then the solutions $u_{s}$ are uniformly Hölder continuous for any exponent $\alpha<2 /(n q+1)$ and the Hölder constant is uniformly controlled by $C$ and the constants from the definition of the uniformly bounded geometry.

As a direct application of Theorem $\mathrm{A}^{*}$ one has the following corollary:

Corollary 3.2. Suppose $X$ is a compact Kähler manifold and $\omega$ is a $\mathcal{C}^{1,1}$ smooth closed positive form on $X$. Suppose moreover that $\omega$ can be approximated in $\mathcal{C}^{1,1}$ - norm by smooth closed forms with curvatures bounded by a fixed constant. Let also $f$ be any nonnegative function such that $f \in L^{p}\left(\omega^{n}\right)$ and $\int_{X} f \omega^{n}=\int_{X} \omega^{n}$. Then the Monge-Ampère equation

$$
\left(\omega+d d^{c} u\right)^{n}=f \omega^{n}, \sup _{X} u=0
$$

has an $\alpha$-Hölder continuous solution $u$ for any $\alpha<2 /(n q+1)$, where $q$ is the conjugate to $p$.

Finally we remark that in [DZ] the stability result holds not only for measures absolutely continuous with respect to the Lebesgue measure, but also for any measure dominated by capacity for $L^{p}$ functions. Observe that in the proof the sole place where we used the assumption that $\mu$ is a measure with density was the application of the Jensen formula in (3.2). Therefore by repeating the above proof one can get the following generalization:

Proposition 3.3. Let $u \in \operatorname{PSH}(X, \omega)$ solve the equation $M A(u)=\mu$ for $\mu$ a probability measure on a compact Kähler manifold $(X, \omega)$. Assume that $\mu$ satisfies the following additional assumptions: 
i) $\mu$ satisfies $\mathcal{H}(\infty)$;

ii) $\left\|\rho_{\delta} \phi-\phi\right\|_{L^{1}(\mu)}=O\left(\delta^{b}\right)$ for some $b>0$.

Then $u$ is Hölder continuous with the exponent depending only on $n$ and $b$.

Examples of such singular measures have been considered in [Hi].

\section{Some Properties of $\operatorname{MAH}(X, \omega)$}

4.1. The one dimensional case. In this section we recall for reader's convenience the classical one dimensional theory of Hölder continuous potentials. We refer to [DS] for more details. It is worthwhile to recall that the problem on Riemann surfaces is linear and hence much easier: analogous statements in the case of planar domains are classical in potential theory.

Proposition 4.1. Let $(X, \omega)$ be a compact Riemann surface. Let also $\mu=\omega+d d^{c} \phi$ be a probability measure on $X$, where $\phi \in P S H(X, \omega)$ and $\mathbb{B}(a, r)$ be the ball (with respect to the metric induced by $\omega)$ centered at point a with radius $r$. The following properties are equivalent:

i) the function $\phi$ is Hölder continuous ;

ii) there exists constants $\alpha, C>0$ such that $\mu(\mathbb{B}(a, r)) \leq C r^{\alpha}$, for all $a \in X$ and $0<r<1$;

iii) there exists $\varepsilon>0$ such that $\exp (-\varepsilon P S H(X, \omega)) \subset L^{1}(\mu)$.

Remark 4.2. As the Laplacian is a linear operator, Proposition 4.1 is actually a local result. It further holds for higher dimensional subharmonic functions. We let the reader check that if $u$ is a subharmonic function in some domain $\Omega \subset \mathbb{R}^{n}$ which contains the origin, and $0<\alpha<1$, then the following are equivalent:

(1) $\sup _{\mathbb{B}(\delta)} u-u(0) \leq C_{1} \delta^{\alpha}$, for some $C_{1}>0$ and $0<\delta<<1$;

(2) $\frac{1}{\operatorname{vol}(\mathbb{B}(\delta))} \int_{\mathbb{B}(\delta)} u(z) d V(z)-u(0) \leq C_{1} \delta^{\alpha}$, where $C_{1}>0, \quad 0<\delta<<1$;

(3) $\int_{\mathbb{B}(\delta)} \Delta u \leq C_{3} \delta^{\alpha+n-2}$, for some $C_{3}>0$ and $0<\delta<<1$.

It classically follows from this observation that any subharmonic function is $\alpha$-Hölder continuous (respectively $\left.\mathcal{C}^{1, \alpha}\right)$ outside a set of arbitrarily small $(n-2+\alpha)$-Hausdorff (respectively $(n-1+\alpha)$-Hausdorff $)$ content.

4.2. Characterization of $M A H(X, \omega)$. Let $\Omega$ be a bounded domain in $\mathbb{C}^{n}$. Analogously to the formula (2.6) for each $u \in P S H(\Omega)$ and $\delta>0$ we set

$$
\check{u}_{\delta}(z)=\frac{1}{v_{2 n} \delta^{2 n}} \int_{\mathbb{B}_{\delta}} u(z+w) d V(w) \quad \text { and } \quad u_{\delta}(z)=\sup _{w \in \mathbb{B}_{\delta}} u(z+w),
$$

for $z \in \Omega_{\delta}=\{z \in \Omega: d(z, \partial \Omega)>\delta\}$. Here

$$
\mathbb{B}_{\delta}=\left\{z \in \mathbb{C}^{n}:\|z\|=\left(\left|z_{1}\right|^{2}+\ldots+\left|z_{n}\right|^{2}\right)^{\frac{1}{2}}<\delta\right\}
$$

and $v_{2 n}$ is the volume of the unit ball $\mathbb{B}_{1}$.

Theorem 4.3. Let $(X, \omega)$ be a compact Kähler manifold, $\mu$ a positive Borel measure on $X$ so that $\mu(X)=\int_{X} \omega^{n}$. The following are equivalent:

i) There exists a Hölder continuous $\omega$-psh $\varphi$ such that $\mu=\left(\omega+d d^{c} \varphi\right)^{n}$.

ii) For every $z \in X$, there exists a neighborhood $D$ of $z$ and a Hölder continuous psh $v$ on $D$ such that $\left.\mu\right|_{D} \leq\left(d d^{c} v\right)^{n}$.

iii) $\mu \in \mathcal{H}(\infty)$ and there exists $C, \alpha>0$ such that $\int_{K}\left[\check{u}_{\delta}-u\right] d \mu \leq C \int_{\bar{D}} \Delta u \delta^{\alpha}$, for all $u \in \mathrm{PSH} \cap L^{\infty}(\Omega), K \subset \subset D \subset \subset \Omega$, where $\Omega$ is a local chart.

A positive measure $\mu$ thus belongs to $\operatorname{MAH}(X, \omega)$ if and only if it is locally the MongeAmpère measure of a Hölder-continuous psh function. 
Proof. The implication i) $\Rightarrow$ ii) is immediate. The implication iii) $\Rightarrow$ i) was observed to hold in Proposition 3.3.

We now consider the implication ii) $\Rightarrow$ iii). It is enough to prove the inequality

$$
\int_{K}\left[\check{u}_{\delta}-u\right]\left(d d^{v}\right)^{n} \leq C \int_{\bar{D}} \Delta u \delta^{\alpha}
$$

for all $u \in \mathrm{PSH} \cap L^{\infty}(\Omega), K \subset \subset D \subset \subset \Omega$ and for any local chart $\Omega$.

We can assume without loss of generality that $K=\mathbb{B}_{1}$ is the unit ball in $\mathbb{C}^{n}, D=\mathbb{B}_{2}$ and $-2 \leq v \leq-1,|v(z)-v(w)| \leq\|z-w\|^{s}$ for all $z, w \in \mathbb{B}_{2}$. This implies that $h(z):=\|z\|^{2}-4<v$ on $\mathbb{B}_{1}$, while $v<h$ on $\mathbb{B}_{2} \backslash \mathbb{B}_{r_{0}}$ for some $1<r_{0}<2$.

Replacing $v$ by $\max (v, h)$ we can assume that $v=h$ on $\mathbb{B}_{2} \backslash \mathbb{B}_{r_{0}}$. Fix $\rho \in C_{0}^{\infty}\left(\mathbb{C}^{n}\right)$ such that $\rho \geq 0, \rho(z)=\rho(\|z\|), \operatorname{supp} \rho \subset \mathbb{B}_{1}$ and $\int_{\mathbb{C}^{n}} \rho(z) d V(z)=1$. Set

$$
\hat{v}_{\delta}(z)=\int_{\mathbb{B}_{1}} v(z-\delta w) \rho(w) d V(w)=\frac{1}{\delta^{2 n}} \int_{\mathbb{B}(z, \delta)} v(w) \rho\left(\frac{z-w}{\delta}\right) d V(w) .
$$

Observe that

$$
\hat{v}_{\delta}(z)-v(z)=\int_{\mathbb{B}_{1}}[v(z-\delta w)-v(z)] \rho(w) d V(w) \leq \delta^{s}
$$

and

$$
\left|\frac{\partial^{2} \hat{v}_{\delta}}{\partial z_{j} \partial \bar{z}_{k}}(z)\right| \leq \frac{C\|v\|_{L^{\infty}(\Omega)}}{\delta^{2}}, \quad\left(d d^{c} \hat{v}_{\delta}\right)^{n} \leq \frac{C d V}{\delta^{2 n}} .
$$

Choose now $\phi \in C_{0}^{\infty}\left(\mathbb{C}^{n}\right)$ such that $0 \leq \phi \leq 1, \phi=1$ on $\mathbb{B}_{r_{1}}$ and $\operatorname{supp} \phi \subset \mathbb{B}_{r_{2}}$, where $r_{0}<r_{1}<r_{2}<2$. Set

$$
\bar{v}_{\delta}(z)=\int_{\mathbb{B}_{1}} v(z-\delta \phi(z) w) \rho(w) d V(w)
$$

Observe that

$$
\bar{v}_{\delta}(z)-v(z)=\int_{\mathbb{B}_{1}}[v(z-\delta \phi(z) w)-v(z)] \rho(w) d V(w) \leq \delta^{s}
$$

and

$$
\bar{v}_{\delta}(z)=\hat{v}_{\delta}(z) \text { on } \mathbb{B}_{r_{1}}, \quad \bar{v}_{\delta}(z)=v(z) \text { on } \mathbb{B}_{2} \backslash \mathbb{B}_{r_{2}} .
$$

Fix now any $z \in \mathbb{B}_{2} \backslash \overline{\mathbb{B}}_{r_{0}}$. Since $v=h$ there, we have for any $\delta<\delta_{0}$,

$$
\begin{aligned}
\frac{\partial^{2} \bar{v}_{\delta}}{\partial z_{j} \partial \bar{z}_{k}}(z) & =\int_{\mathbb{B}_{1}}\left[\frac{\partial^{2} h}{\partial z_{j} \partial \bar{z}_{k}}(z-\delta \phi(z) w)+\delta O(1)\right] \rho(w) d V(w) \\
& =\int_{\mathbb{B}_{1}}\left[\delta_{j k}+\delta O(1)\right] \rho(w) d V(w) \\
& =\delta_{j k}+\delta O(1) .
\end{aligned}
$$

Therefore $\bar{v}_{\delta} \in \operatorname{PSH}\left(\mathbb{B}_{2} \backslash \overline{\mathbb{B}}_{r_{0}}\right), \forall \delta<\delta_{0}$, hence $\bar{v}_{\delta}$ is actually plurisubharmonic in all of $\mathbb{B}_{2}$ (if $\delta$ is small enough), as follows from (4). Set

$$
T:=\sum_{j=0}^{n-1}\left(d d^{c} v\right)^{j} \wedge\left(d d^{c} \bar{v}_{\delta^{\varepsilon}}\right)^{n-1-j} .
$$


¿From (3), (4) and Stokes fomula we get

$$
\begin{aligned}
\int_{K}\left[\check{u}_{\delta}-u\right]\left(d d^{c} v\right)^{n} & \leq \int_{\mathbb{B}_{2}}\left[\check{u}_{\delta}-u\right]\left(d d^{c} v\right)^{n} \\
& =\int_{\mathbb{B}_{2}}\left[\check{u}_{\delta}-u\right]\left[\left(d d^{c} v\right)^{n}-\left(d d^{c} \bar{v}_{\delta^{\varepsilon}}\right)^{n}\right]+\int_{\mathbb{B}_{2}}\left[\check{u}_{\delta}-u\right]\left(d d^{c} \bar{v}_{\delta^{\varepsilon}}\right)^{n} \\
& \leq \int_{\mathbb{B}_{2}}\left[\check{u}_{\delta}-u\right] d d^{c}\left(v-\bar{v}_{\delta^{\varepsilon}}\right) \wedge T+\frac{C}{\delta^{2 n \varepsilon}} \int_{\mathbb{B}_{2}}\left[\check{u}_{\delta}-u\right] d V \\
& \leq \int_{\mathbb{B}_{2}}\left[\bar{v}_{\delta^{\varepsilon}}-v\right] d d^{c}\left(u-\check{u}_{\delta}\right) \wedge T+\frac{C \int_{\mathbb{B}_{2}} \Delta u \delta^{2}}{\delta^{2 n \varepsilon}} \\
& \leq \int_{\mathbb{B}_{2}}\left[\bar{v}_{\delta^{\varepsilon}}-v\right] d d^{c} u \wedge T+C \int_{\mathbb{B}_{2}} \Delta u \delta^{2(1-n \varepsilon)} \\
& \leq \delta^{\varepsilon s} \int_{\mathbb{B}_{r_{2}}} d d^{c} u \wedge T+C \delta^{2(1-n \varepsilon)} \int_{\mathbb{B}_{2}} \Delta u \\
& \leq C\left[\delta^{\varepsilon s}\|v\|_{L^{\infty}(\Omega)}^{n-1} \int_{\mathbb{B}_{2}} \Delta u+\delta^{2(1-n \varepsilon)} \int_{\mathbb{B}_{2}} \Delta u\right] \\
& \leq C \int_{\mathbb{B}_{2}} \Delta u \delta^{\alpha},
\end{aligned}
$$

where $\varepsilon=\frac{2}{s+2 n}, \alpha=\frac{2 s}{s+2 n}$.

4.3. Proof of Theorem B. Below we derive several simple consequences of this characterization. First, the range of the complex Monge-Ampère operator has the " $L^{p}$-property":

Corollary 4.4. Let $\psi \in \operatorname{PSH}(X, \omega)$ be a Hölder continuous function. Consider a density $0 \leq f \in L^{p}\left(\left(\omega+d d^{c} \psi\right)^{n}\right)$ with $p>1$ and $\int_{X} f\left(\omega+d d^{c} \psi\right)^{n}=\int_{X} \omega^{n}$. Then there exists a Hölder continuous $\omega$-plurisubharmonic function $\varphi$ such that

$$
\left(\omega+d d^{c} \varphi\right)^{n}=f\left(\omega+d d^{c} \psi\right)^{n} .
$$

In particular $\operatorname{MAH}(X, \omega)$ is a convex set.

Proof. By Hölder inequality we have

$$
\int_{K} f \omega_{\psi}^{n} \leq\|f\|_{L^{p}\left(\omega_{\psi}^{n}\right)}\left[\omega_{\psi}^{n}(K)\right]^{1-\frac{1}{p}},
$$

for any Borel subset $K$ of $X$. This implies that $f \omega_{\psi}^{n} \in \mathcal{H}(\infty)$. On the other hand, by Hölder inequality we have

$$
\int_{K}\left[\check{u}_{\delta}-u\right] f \omega_{\psi}^{n} \leq\|f\|_{L^{p}\left(\omega_{\psi}^{n}\right)}\left[\int_{K}\left[\check{u}_{\delta}-u\right] \omega_{\psi}^{n}\right]^{1-\frac{1}{p}} \leq C \delta^{\alpha},
$$

for all $u \in \operatorname{PSH} \cap L^{\infty}(\Omega), K \subset \subset D \subset \subset \Omega$ and local chart $\Omega$. Therefore using Theorem 2.1 there exists a Hölder continuous $\omega$-psh function $\varphi$ such that $\omega_{\varphi}^{n}=f \omega_{\psi}^{n}$.

Fix $\mu_{1}=\operatorname{MA}\left(\phi_{1}\right), \mu_{2}=\operatorname{MA}\left(\phi_{2}\right) \in \operatorname{MAH}(X, \omega)$ and set $\mu=\left(\mu_{1}+\mu_{2}\right) / 2$. Observe that $\psi:=\left(\phi_{1}+\phi_{2}\right) / 2 \in \operatorname{PSH}(X, \omega) \cap \operatorname{Hölder}(X, \omega)$ satisfies

$$
\left(\omega+d d^{c} \psi\right)^{n} \geq \frac{1}{2^{n}}\left(\mu_{1}+\mu_{2}\right)
$$

hence $\mu=f\left(\omega+d d^{c} \psi\right)^{n}$ with bounded density $0 \leq f \leq 2^{n-1}$. It therefore follows from the first part of the corollary that $\mu$ also belongs to $\operatorname{MAH}(X, \omega)$, hence the latter is convex.

We also note that the range of the complex Monge-Ampère operator has the product property. 
Corollary 4.5. Let $\left(X_{1}, \omega_{1}\right),\left(X_{2}, \omega_{2}\right)$ be two compact Kähler manifolds of dimension $n_{1}, n_{2}$, normalized so that $\int_{X_{1}} \omega_{1}^{n_{1}}=\int_{X_{2}} \omega_{2}^{n_{2}}=1$. Fix $\mu_{1}, \mu_{2}$ two probability measures on $X_{1}, X_{2}$. The following are equivalent:

i) $\mu_{1} \in \operatorname{MAH}\left(X_{1}, \omega_{1}\right)$ and $\mu_{2} \in \operatorname{MAH}\left(X_{2}, \omega_{2}\right)$.

ii) $\mu=\mu_{1} \times \mu_{2} \in \operatorname{MAH}\left(X_{1} \times X_{2}, \omega\right)$, where

$$
\omega=\left(\begin{array}{c}
n_{1}+n_{2} \\
n_{1}
\end{array}\right)^{-1 /\left(n_{1}+n_{2}\right)}\left[\omega_{1}+\omega_{2}\right]
$$

Here $\mu=\mu_{1} \times \mu_{2}$ denotes the product (probability) measure on $X_{1} \times X_{2}$, and we still denote by $\omega_{1}, \omega_{2}$ the semi-positive forms on $X_{1} \times X_{2}$ obtained by pulling-back $\omega_{1}, \omega_{2}$ on each factor.

Proof. i) $\Rightarrow$ ii) Assume that $\mu_{1}=\left(\omega_{1}+d d^{c} u_{1}\right)^{n_{1}}$ and $\mu_{2}=\left(\omega_{2}+d d^{c} u_{2}\right)^{n_{2}}$ where $u_{1}, u_{2}$ are Hölder continuous $\omega_{i}$-psh functions on $X_{1}, X_{2}$. Pulling back these forms and functions on $X=X_{1} \times X_{2}$ and observing that $\left(\omega_{i}+d d^{c} u_{i}\right)^{1+n_{i}} \equiv 0$, one obtains

$$
\mu=\mu_{1} \times \mu_{2}=\left(\omega+d d^{c} u\right)^{n_{1}+n_{2}} \text { with } u=\frac{\left[u_{1}+u_{2}\right]}{\left(\begin{array}{c}
n_{1}+n_{2} \\
n_{1}
\end{array}\right)^{1 /\left(n_{1}+n_{2}\right)}}
$$

so that $\mu \in \operatorname{MAH}(X, \omega)$.

ii) $\Rightarrow$ i) Since $\mu$ satisfies iii) in Theorem 4.3 we infer that $\mu_{1}, \mu_{2}$ satisfy the same property. Using Theorem 4.3 again thus yields $\mu_{1} \in \operatorname{MAH}\left(X_{1}, \omega_{1}\right), \mu_{2} \in \operatorname{MAH}\left(X_{2}, \omega_{2}\right)$.

\section{Measures With Symmetries}

Generalizing Skoda's celebrated result [Sk], Dinh-Nguyen-Sibony have observed recently [DNS] that if $\mu$ is the Monge-Ampère measure of a Hölder-continuous quasi-psh function, then

$$
\exp (-\varepsilon \operatorname{PSH}(X, \omega)) \subset L^{1}(\mu)
$$

for $\varepsilon>0$ small enough. We show here that the converse holds when $\mu$ moreover has radial or toric singularities. The general case is open, see however [Hi] for some partial results.

5.1. Exponential integrability, Lelong numbers and symmetries- basic results. Note for later use that if $\exp (-\varepsilon \operatorname{PSH}(X, \omega)) \subset L^{1}(\mu)$, then for all $x \in X$ and $0<r<<1$,

$$
\mu(\mathbb{B}(x, r)) \leq C r^{\varepsilon}
$$

and $\mu(K) \leq C T(K)^{\varepsilon}$ for all Borel sets $K$, where $T$ denote the Alexander-Taylor capacity (see [GZ1]). This implies that for all $A>1$, there exists $C_{A}>0$ such that

$$
\mu(K) \leq C_{A} \operatorname{Cap}_{\omega}(K)^{A}, \text { for all Borel set } K,
$$

where $\operatorname{Cap}_{\omega}$ denotes the Monge-Ampère capacity. In other words, $\mu$ is very well dominated by the Monge-Ampère capacity (it satisfies the condition $\mathcal{H}(\infty)$ ).

Let $u$ be a psh function defined near the origin in $\mathbb{C}^{n}$, with a radial singularity, i.e. such that $u(z)=u(\|z\|)$ for all $z$. It is then standard that $u$ can be written as $u(z)=\chi \circ L(z)$ where $L(z)=\log \|z\|$ and $\chi$ is a convex increasing function defined in a neighborhood of $-\infty$. Note that

- the function $u$ is bounded if and only $\chi(-\infty)>-\infty$;

- the Lelong number $\nu(u, 0)$ is non zero if and only if $\chi(t) \sim \nu(u, 0) t$ near $-\infty$, which is the maximal growth that $\chi$ can have at $-\infty$. Alternatively, $\nu(u, 0)=0$ if and only if $\chi^{\prime}(-\infty)=0$. The following elementary computation is left to the reader: 
Lemma 5.1. Let $u=\chi \circ L$ be a radial plurisubharmonic function defined in a ball $\mathbb{B} \ni 0$. Assume that $\chi$ is $\mathcal{C}^{2}$ smooth. Then $u$ belongs to the domain of definition of the Monge-Ampère operator and

$$
\left(d d^{c} u\right)^{n}=\nu(u, 0)^{n} \delta_{0}+c_{n}\left(\chi^{\prime} \circ L\right)^{n-1} \chi^{\prime \prime} \circ L \frac{d V}{\|z\|^{2 n}} .
$$

Here $\delta_{0}$ denotes the Dirac mass at the origin. Note in particular that when $\nu(u, 0)=0$ then the Monge-Ampère measure $\left(d d^{c} u\right)^{n}$ is absolutely continuous with respect to Lebesgue measure.

A similar formula can be derived for Monge-Ampère measures with toric symmetries, but we will not use it: we will handle the toric case by using Theorem 4.3, whereas the radial case will be treated directly, using Lemma 5.1 (the direct method yields better exponents).

5.2. The radial case. We obtain here a complete description of those radial measures which belong to $\operatorname{MAH}(X, \omega)$.

Proposition 5.2. Let $\mu$ be a probability measure on $X$ which is smooth but at finitely many points where it has a radial singularity. The following are equivalent:

i) $\exp (-\varepsilon \operatorname{PSH}(X, \omega)) \subset L^{1}(\mu)$ for all $0<\varepsilon<\varepsilon_{0}$;

ii) $\|z-a\|^{-\varepsilon} \in L^{1}(\mu)$ for all $0<\varepsilon<\varepsilon_{0}$ and $a \in X$;

iii) $\mu(\mathbb{B}(a, r)) \leq C r^{\varepsilon}$ for all $0<\varepsilon<\varepsilon_{0}$ and $a \in X$;

iv) $\mu=\left(\omega+d d^{c} \phi\right)^{n}$, where $\phi \in \operatorname{PSH}(X, \omega)$ is Hölder continuous with exponent $\alpha$ arbitrarily close to $\varepsilon_{0} / n$.

Proof. The implication i) $\Rightarrow$ ii) is obvious. The equivalence ii) $\Leftrightarrow$ iii) is immediate. The implication iv) $\Rightarrow$ iii) is classical (successive integration by parts against a cut-off function with support in a corona of radii $j r,(j+1) r)$ and holds for general (non radial) measures. The implication iv) $\Rightarrow \mathrm{i}$ ) was obtained in [DNS], also for general measures. In the sequel we thus focus on the remaining implication ii) $\Rightarrow$ iv).

Let $a \in X$ be one of the finitely many singular points. We fix a local chart near $a$ such that $a=0$ is the origin and locally $\mu=\left(d d^{c} u\right)^{n}$ with $u=\chi \circ L, L(z)=\log \|z\|$ and $\chi$ convex increasing. Observe that $u$ is bounded and $\chi^{\prime}(-\infty)=0$. By Theorem 4.3 it is enough to check that $u$ is Hölder continuous at point $a$, which is equivalent to showing that

$$
0 \leq \chi(t)-\chi(-\infty) \leq C \exp (\delta t) \text { as } t \rightarrow-\infty,
$$

for some positive constants $C, \delta>0$.

By assumption there exists $\varepsilon>0$ such that $\|z\|^{-\varepsilon} \in L^{1}(\mu)$. We infer from Lemma 5.1 that

$$
\begin{gathered}
\int_{0} \frac{1}{\|z\|^{\varepsilon}} d \mu=c \int_{0}\left(\chi^{\prime} \circ L\right)^{n-1} \chi^{\prime \prime} \circ L \frac{d V(z)}{\|z\|^{2 n+\varepsilon}} \\
=c^{\prime} \int_{-\infty}\left(\chi^{\prime}(t)\right)^{n-1} \chi^{\prime \prime}(t) e^{-\varepsilon t} d t<+\infty .
\end{gathered}
$$

We now integrate by parts, in finite time, to obtain

$$
\varepsilon \int_{-A}\left(\chi^{\prime}\right)^{n} \exp (-\varepsilon t) d t=\left(\chi^{\prime}\right)^{n}(-A) \exp (+\varepsilon A)+O(1)
$$

We claim that $\int_{-\infty}\left(\chi^{\prime}\right)^{n} \exp (-\varepsilon t) d t$ is finite. So is the limsup on the right hand side, hence $\chi^{\prime}(t) \leq C \exp (\varepsilon t / n)$, which yields

$$
\chi(t)-\chi(-\infty) \leq C^{\prime} \exp (\varepsilon t / n) .
$$

Therefore $u(z)-u(0) \leq C^{\prime}\|z\|^{\varepsilon / n}$, i.e. $u$ is Hölder continuous. 
It remains to prove the claim. If $\int_{-\infty}\left(\chi^{\prime}\right)^{n} \exp (-\varepsilon t) d t=+\infty$, then $\left(\chi^{\prime}\right)^{n}(-A) \exp (+\varepsilon A) \rightarrow+\infty$ as $A \rightarrow+\infty$. Set

$$
h(t)=\left(\chi^{\prime}\right)^{n}(t) \exp (-\varepsilon t) \text { and } H(x)=\int_{x}^{0} h(t) d t .
$$

Thus $H(x) \rightarrow+\infty$ as $x \rightarrow-\infty$ and

$$
\varepsilon H(x)=H^{\prime}(x)+O(1) .
$$

We let the reader check that this implies $H(x)=\lambda \exp (-\varepsilon x)+O(1)$ for some constant $\lambda \geq 0$. Now $\chi^{\prime}(t) \rightarrow 0$ as $t \rightarrow-\infty$ so $h(t)=o(\exp (-\varepsilon t))$ and $H(t)=o(\exp (-\varepsilon t))$. This forces $\lambda=0$, hence $H(t)=O(1)$.

5.3. The toric case. We now consider the case of probability measures $\mu$ which are smooth but at finitely many points where they have "toric singularities" the origin $0 \in \mathbb{C}^{n}$ is called a toric singularity for the measure $\mu=\left(d d^{c} u\right)^{n}, u$ psh and bounded, if $u$ is $\left(S^{1}\right)^{n}$-invariant, i.e.

$$
u\left(z_{1}, \ldots, z_{n}\right)=u\left(\left|z_{1}\right|, \ldots,\left|z_{n}\right|\right), \quad \forall z=\left(z_{1}, \ldots, z_{n}\right) \in \Delta^{n} .
$$

We will call these measures toric measures for short.

Proposition 5.3. Let $\mu$ be a toric measure in the unit polydisk $\Delta^{n} \subset \mathbb{C}^{n}$. Assume that for all $0<r<\frac{1}{2}$ and $j=1, \ldots, n$,

$$
\mu\left(\Delta \times \ldots \times \Delta_{j}(r) \times \ldots \times \Delta\right) \leq C r^{\alpha}, \text { where } C, \alpha>0 .
$$

Then

$$
\int_{\Delta_{n}(t)}\left[\check{u}_{\delta}(z)-u(z)\right] d \mu \leq C(t) \delta^{\beta}
$$

for all $0<t<1$ and $u \in \operatorname{PSH} \cap L^{\infty}\left(\Delta^{n}\right)$ with $0 \leq u \leq 1$.

Proof. Set $T u(z)=\frac{1}{(2 \pi)^{n}} \int_{[0,2 \pi]^{n}} u\left(e^{i \theta_{1}}\left|z_{1}\right|, \ldots, e^{i \theta_{n}}\left|z_{n}\right|\right) d \theta_{1} \ldots d \theta_{n}$. Note that

$T u(z)=T u\left(\left|z_{1}\right|, \ldots,\left|z_{n}\right|\right)$ is increasing and logarithmically convex. This implies that

$$
T u\left(\left|z_{1}\right|+\delta_{1}, \ldots,\left|z_{n}\right|+\delta_{n}\right)-T u\left(\left|z_{1}\right|, \ldots,\left|z_{n}\right|\right) \mid \leq C \sum_{j=1}^{n} \log \left(1+\frac{\delta_{j}}{\left|z_{j}\right|}\right),
$$

for all $z \in \Delta_{n}(1 / 2)$. It follows from Fubini theorem that

$$
\begin{aligned}
T \check{u}_{\delta}(z) & =\frac{1}{(2 \pi)^{n}} \int_{[0,2 \pi]^{n}} \check{u}_{\delta}\left(e^{i \theta_{1}}\left|z_{1}\right|, \ldots, e^{i \theta_{n}}\left|z_{n}\right|\right) d \theta_{1} \ldots d \theta_{n} \\
& =\frac{1}{(2 \pi)^{n}} \int_{[0,2 \pi]^{n}} \frac{1}{c_{n} \delta^{n}} \int_{\mathbb{B}_{\delta}} u\left(e^{i \theta_{1}}\left|z_{1}\right|+w_{1}, \ldots, e^{i \theta_{n}}\left|z_{n}\right|+w_{n}\right) d V(w) d \theta_{1} \ldots d \theta_{n} \\
& =\frac{1}{(2 \pi)^{n}} \int_{[0,2 \pi]^{n}} \frac{1}{c_{n} \delta^{n}} \int_{\mathbb{B}_{\delta}\left(\left|z_{1}\right|, \ldots,\left|z_{n}\right|\right)} u\left(e^{i \theta_{1}} \xi_{1}, \ldots, e^{i \theta_{n}} \xi_{n}\right) d V(\xi) d \theta_{1} \ldots d \theta_{n} \\
& =\frac{1}{c_{n} \delta^{n}} \int_{\mathbb{B}_{\delta}\left(\left|z_{1}\right|, \ldots,\left|z_{n}\right|\right)} T u(\xi) d V(\xi) \\
& \leq T u\left(\left|z_{1}\right|+\delta, \ldots,\left|z_{n}\right|+\delta\right) .
\end{aligned}
$$

Since $\mu$ is toric,

$$
\int_{\Delta_{n}(1 / 2)}\left[\check{u}_{\delta}(z)-u(z)\right] d \mu=\int_{\Delta_{n}(1 / 2)}\left[T \check{u}_{\delta}(z)-T u(z)\right] d \mu
$$


thus

$$
\begin{aligned}
\int_{\Delta_{n}(1 / 2)}\left[\check{u}_{\delta}(z)-u(z)\right] d \mu & \leq C \sum_{j=1}^{n} \int_{\Delta_{n}(1 / 2)} \log \left(1+\frac{\delta}{\left|z_{j}\right|}\right) d \mu \\
& \leq n C \int_{0}^{1 / 2} \frac{\delta t^{\alpha}}{t^{2}+\delta t} d t \\
& \leq C^{\prime} \delta^{\beta}
\end{aligned}
$$

with $\beta=\alpha /(\alpha+2)$, as can be checked by cutting the integral into two pieces $\int_{0}^{\delta^{\gamma}}+\int_{\delta^{\gamma}}^{1 / 2}$, where $\gamma=1 /(\alpha+2)$.

Corollary 5.4. A toric probability measure $\mu$ belongs to $\operatorname{MAH}(X, \omega)$ if and only if $\exp (-\varepsilon \operatorname{PSH}(X, \omega)) \subset L^{1}(\mu)$ for some $\varepsilon>0$.

Proof. If $\mu$ belongs to $\operatorname{MAH}(X, \omega)$, then $\exp (-\varepsilon \operatorname{PSH}(X, \omega)) \subset L^{1}(\mu)$ for some $\varepsilon>0$, as follows from [DNS]. Assume now that $\exp (-\varepsilon \operatorname{PSH}(X, \omega)) \subset L^{1}(\mu)$ for some $\varepsilon>0$. As explained earlier, this implies that $\mu$ is very well dominated by the Monge-Ampère capacity, in particular $\mu \in \mathcal{H}(\infty)$. The previous proposition shows that item (iii) of Theorem 4.3 applies, hence $\mu \in \operatorname{MAH}(X, \omega)$.

In view of the above proofs, one may wonder whether all probability measures satisfying condition $\mathcal{H}(\infty)$ belong to $\operatorname{MAH}(X, \omega)$. The following example shows this is far from being the case.

Example 5.5. We assume here $(X, \omega)=\left(\mathbb{P}^{1}, \omega_{F S}\right)$ is the Riemann sphere equipped with the Fubini-Study form. We let $\phi \in \operatorname{PSH}(X, \omega)$ be a function that is smooth in $\mathbb{P}^{1}$ but at one point which we choose as the origin 0 in some affine chart $\mathbb{C}$ and so that

$$
\phi(z)=\exp (-\sqrt{-\log |z|})-\frac{1}{2} \log \left[1+|z|^{2}\right]
$$

near the origin. The reader will easily check, following the arguments in Example 4.2 in [BGZ], that $\mu=\omega+d d^{c} \phi$ is very well dominated by the logarithmic capacity, in particular satisfies $\mathcal{H}(\infty)$, although $\phi$ is not Hölder continuous.

\section{The CASE of Big COHOMOlOGY ClASSES}

Proof of Theorem D. In order to deal with the general case of big cohomology classes, we use again the regularization techniques of the first author, coupled now with Proposition 2.9.

We let $\varphi$ be a $\theta$-psh function solution of $\left(\theta+d d^{c} \varphi\right)^{n}=\mu$, where the density $f \geq 0$ of $\mu$ with respect to a smooth volume form belongs to $L^{p}$ for some $p>1$. The solution is unique up to an additive constant, it is $\theta$-psh with minimal singularities (see [BEGZ]). We can thus assume, without loss of generality, that $-C_{0}+V_{\theta} \leq \varphi \leq V_{\theta}$. We let

$$
\varphi \mapsto \rho_{\delta} \varphi
$$

again denote the regularization operator defined in (2.1). As in the Kähler case $t \mapsto \rho_{t} \varphi+K t^{2}$ is increasing for $0 \leq t \leq \delta_{0}$ and some constant $K$.

We consider the Kiselman-Legendre transform,

$$
\psi_{c, \delta}(z):=\inf _{t \in] 0, \delta]}\left\{\rho_{t} \varphi(z)+K t^{2}-c \log (t / \delta)\right\},
$$

where $0 \leq \delta \leq \delta_{0}$ and $c>0$ will be carefully chosen below. Observe that

$$
\varphi \leq \psi_{c, \delta} \leq \rho_{\delta} \varphi+K \delta^{2} .
$$

The fundamental curvature estimate is now

$$
\theta+d d^{c} \psi_{c, \delta} \geq-\left(A c+K \delta^{2}\right) \omega
$$


for some constant $A>0$. Since the coholomogy class $\Theta=\{\theta\}$ is big, there exists a $\theta$-psh function $\psi_{0}$ on $X$ such that $\theta+d d^{c} \psi_{0} \geq \varepsilon_{0} \omega$, for some small constant $\varepsilon_{0}>0$. Subtracting a large constant, we can always assume that $\psi_{0} \leq 0$ hence $\psi_{0} \leq V_{\theta}$.

It follows that the function

$$
\varphi_{c, \delta}:=\frac{A c+K \delta^{2}}{\varepsilon_{0}} \psi_{0}+\left(1-\frac{A c+K \delta^{2}}{\varepsilon_{0}}\right) \psi_{c, \delta}
$$

is $\theta$-plurisubharmonic on $X$. Fix $0<\delta<\delta_{0}$ and choose $c>0$ such that

$$
A c+K \delta^{2}=\varepsilon_{0} \delta^{\alpha}, \text { where } \alpha:=2 \gamma,
$$

and observe that $c=\varepsilon_{0} A^{-1} \delta^{\alpha}-K A^{-1} \delta^{2}=O\left(\delta^{\alpha}\right)$. In the sequel we set

$$
\varphi_{\delta}:=\varphi_{c, \delta}
$$

Since $\psi_{0} \leq V_{\theta} \leq \varphi+C_{0}$, we see from the definition that on the ample locus,

$$
\begin{aligned}
\varphi_{\delta}-\varphi & =\delta^{\alpha}\left(\psi_{0}-\varphi\right)+\left(1-\delta^{\alpha}\right)\left(\psi_{c, \delta}-\varphi\right) \\
& \leq C_{0} \delta^{\alpha}+\left(1-\delta^{\alpha}\right)\left(\rho_{\delta} \varphi-\varphi+K \delta^{2}\right) .
\end{aligned}
$$

Furthermore, since $\varphi \leq V_{\theta} \leq 0$, we get $\varrho_{\delta} \varphi \leq 0$, thus $\psi_{c, \delta} \leq K \delta^{2} \leq C_{0} \delta^{\alpha}$ if $\delta \leq \delta_{0}$ small enough, and so $\varphi_{\delta} \leq C_{0} \delta^{\alpha}$. This implies $\psi:=\varphi_{\delta}-C_{0} \delta^{\alpha} \leq V_{\theta}$. By Proposition 2.9, it follows that

$$
\begin{aligned}
\sup _{X}\left(\varphi_{\delta}-\varphi\right) & \leq B_{0}\left\|\max \left(\varphi_{\delta}-\varphi-C_{0} \delta^{\alpha}, 0\right)\right\|_{L^{1}(X)}^{\gamma}+C_{0} \delta^{\alpha} \\
& \leq B_{0}\left\|\rho_{\delta} \varphi+K \delta^{2}-\varphi\right\|_{L^{1}(X)}^{\gamma}+C_{0} \delta^{\alpha}
\end{aligned}
$$

for some constant $B_{0}>0$ which depends only on $\gamma$ and the uniform norm of $\varphi-V_{\theta}$.

Applying Lemma 2.3, the last estimate yields

$$
\sup _{X}\left(\varphi_{\delta}-\varphi\right) \leq C_{1} \delta^{\alpha}
$$

where $C_{1}:=B_{0} C_{\omega}+K^{\gamma}+C_{0}$ and $C_{\omega}$ is the constant in Lemma 2.3.

This inequality $\varphi_{\delta} \leq \varphi+C_{1} \delta^{\alpha}$ yields a uniform lower bound on the parameter $t=t(z)$ which realizes the infimum in the definition of $\varphi_{\delta}(z)$ for a fixed $z \in \Omega$. Namely the last inequality gives

$$
\begin{aligned}
\varphi_{\delta}(z)-\varphi(z) & =\delta^{\alpha}\left(\psi_{0}(z)-\varphi(z)\right)+\left(1-\delta^{\alpha}\right)\left(\rho_{t} \varphi(z)+K t^{2}-\varphi(z)-c \log (t / \delta)\right) \\
& \leq C_{1} \delta^{\alpha} .
\end{aligned}
$$

Since $V_{\theta}-\varphi \geq 0$ and $\rho_{t} \varphi(z)+K t^{2}-\varphi(z) \geq 0$, it follows that

$$
c\left(1-\delta^{\alpha}\right) \log [t(z) / \delta] \geq \delta^{\alpha}\left(\psi_{0}(z)-V_{\theta}(z)-C_{1}\right) .
$$

Since $c=\varepsilon_{0} A^{-1} \delta^{\alpha}-K A^{-1} \delta^{2}$, the choice $\delta \leq \delta_{1}:=\min \left\{\delta_{0},\left(\varepsilon_{0} / 2 K\right)^{1 /(2-\alpha)}\right\}$ yields $c \geq \frac{1}{2} \varepsilon_{0} A^{-1} \delta^{\alpha}$ and therefore

$$
t(z) \geq \delta \kappa(z)
$$

where

$$
\begin{gathered}
\kappa(z):=\exp \left(C_{2}\left(\psi_{0}(z)-V_{\theta}(z)-C_{1}\right),\right. \\
C_{2}:=\frac{2 A}{\varepsilon_{0}\left(1-\delta_{0}^{\alpha}\right)} .
\end{gathered}
$$

We are now in position to conclude. Fix $z \in A m p(\Theta)$. Since $t(z) \geq \kappa(z) \delta$ and $t \mapsto \rho_{t} \varphi+K t^{2}$ is increasing, we get

$$
\begin{aligned}
\rho_{\kappa(z) \delta} \varphi(z)-\varphi(z) & \leq \rho_{t(z)} \varphi(z)+K t(z)^{2}-\varphi(z) \\
& =\psi_{c, \delta}(z)-\varphi(z)=\frac{1}{1-\delta^{\alpha}}\left(\varphi_{\delta}(z)-\delta^{\alpha} \psi_{0}(z)\right)
\end{aligned}
$$


and by the above and the assumption $\varphi \leq V_{\theta} \leq 0$ we find

$$
\begin{aligned}
\varphi_{\delta}-\delta^{\alpha} \psi_{0} & \leq \varphi+C_{1} \delta^{\alpha}-\delta^{\alpha} \psi_{0} \leq C_{1} \delta^{\alpha}+\delta^{\alpha}\left(V_{\theta}-\psi_{0}\right), \\
\rho_{\kappa(z) \delta} \varphi(z)-\varphi(z) & \left.\leq\left(1-\delta_{0}^{\alpha}\right)^{-1}\right) \delta^{\alpha}\left(C_{1}+V_{\theta}(z)-\psi_{0}(z)\right) .
\end{aligned}
$$

Replacing $\delta$ by $\kappa(z)^{-1} \delta$ and using (6.1), we obtain for $\delta \leq \delta_{0} \kappa(z)$,

$$
\begin{aligned}
\rho_{\delta} \varphi(z)-\varphi(z) & \leq\left(1-\delta_{0}^{\alpha}\right)^{-1} \delta^{\alpha}\left(C_{1}+V_{\theta}(z)-\psi_{0}(z)\right) \cdot \exp \left(\alpha C_{2}\left(C_{1}+V_{\theta}(z)-\psi_{0}(z)\right)\right) \\
& \leq C_{3} \exp \left(2 \alpha C_{2}\left(C_{1}+V_{\theta}(z)-\psi_{0}(z)\right)\right),
\end{aligned}
$$

where

$$
C_{3}:=\left(\alpha C_{2}\right)^{-1}\left(1-\delta_{0}^{\alpha}\right)^{-1}
$$

This finishes the proof of Theorem $\mathrm{D}$, since $\psi_{0}(z)-V_{\theta}(z)$ is locally bounded from below on $\operatorname{Amp}(\Theta)$ as well as $\kappa(z)$ given by (6.1).

\section{APPENDIX}

We briefly explain below how bounds on the curvature may be used to control the differential of the exponential mapping. This is essentially a variation on the theme of Jacobi vector fields.

Estimates for the differential of the exponential. For accurate computations with the exponential we need to control its differential in terms of the curvature. To this end we determine the Jacobi equations which calculate the variation of geodesics.

Let namely $u \rightarrow u+v$ be a small perturbation of the geodesic $t \rightarrow u(t)$ with initial velocity $\zeta$. Its linearization satisfies

$$
\frac{d^{2} v_{m}}{d t^{2}}=\sum_{j, k, l} R_{j \bar{k} l \bar{m}} \bar{v}_{k} \frac{d u_{j}}{d t} \frac{d u_{l}}{d t}+O(|u(t)|) .
$$

Moreover if $D$ denotes the Levi-Civita connection with respect to $\omega$ then along the geodesic $u(t)$ one can compute

$$
\begin{gathered}
\left(\frac{D \zeta}{d t}\right)_{m}=\frac{d \zeta_{m}}{d t}-\sum_{j, k, l} R_{j \bar{k} l \bar{m}} \frac{d u_{j}}{d t} \zeta_{l}+O\left(|u(t)|^{2}\right) \zeta \\
\left(\frac{D^{2} \zeta(t)}{d t^{2}}\right)_{m}=\frac{d^{2} \zeta_{m}}{d t^{2}}-\sum_{j, k, l} R_{j \bar{k} l \bar{m}} \frac{d \bar{u}_{k}}{d t} \frac{d u_{j}}{d t} \zeta_{l}+O(|u(t)|) \zeta .
\end{gathered}
$$

Let us now put $\zeta=v$. Then the Jacobi equation takes the intrinsic form

$$
\left(\frac{D^{2} v(t)}{d t^{2}}\right)_{m}=\sum_{j, k, l} R_{j \bar{k} l \bar{m}} \bar{v}_{k} \frac{d u_{j}}{d t} \frac{d u_{l}}{d t}-\sum_{j, k, l} R_{j \bar{k} l \bar{m}} \frac{d \bar{u}_{k}}{d t} \frac{d u_{j}}{d t} v_{l} .
$$

In particular the formula holds at $\zeta:=u^{\prime}(0)$. Thus if the curvature is bounded by the constant $R_{0}^{2}$ (the square being taken for the ease of notation), then

$$
\left|\left(D_{v(t)} D_{v(t)}\right)\right| \leq 2 R_{0}^{2}|\zeta|^{2}|v|
$$

This is a vector analogue of the scalar equation $y^{\prime \prime}=2 p y$. By Gronwall's lemma the solution to the corresponding Cauchy problem with data $v(0)=v_{0}, D v(0)=v_{1}$ is estimated by

$$
|v(t)| \leq\left|v_{0}\right| \cosh \left(\sqrt{2} R_{0}|\zeta| t\right)+\frac{\left|v_{1}\right|}{\sqrt{2} R_{0}|\zeta|} \sinh \left(\sqrt{2} R_{0}|\zeta| t\right) .
$$


Let us denote by $\tau_{z, \zeta}(t): T_{Z} X \rightarrow T_{\exp _{z}(t \zeta)} X$ the parallel translation along the geodesic. Let also $\tilde{v}(t):=\tau_{z, \zeta}(t)^{-1} v(t) \in T_{z} X$. Then $\tilde{v}$ satisfies the analogous equation with curvature transported back to $T_{z} X$. Thus

$$
\left|\tilde{v}(t)-v_{0}-v_{1} t\right| \leq\left|v_{0}\right| \cosh \left(\sqrt{2} R_{0}|\zeta| t\right)+\frac{\left|v_{1}\right|}{\sqrt{2} R_{0}|\zeta|} \sinh \left(\sqrt{2} R_{0}|\zeta| t\right)-\left|v_{0}\right|-\left|v_{1}\right| t .
$$

The differential of the ordinary exponential mapping evaluated at $(h, \eta) \in T(T X)_{(z, \xi)} \simeq$ $T_{z} X \otimes T_{z} X$ is precisely $v(1)$ for the solution of the Cauchy problem $v(0)=h, D v(0)=\eta$. Thus (6.8) gives us the bound

$$
\left|\tau_{z, \zeta}(1)^{-1} d \exp _{z}(\zeta)(h, \eta)-(h+\eta)\right| \leq h \cosh \left(\sqrt{2} R_{0}|\zeta|\right)+\frac{\eta}{\sqrt{2} R_{0}|\zeta|} \sinh \left(\sqrt{2} R_{0}|\zeta|\right)-h-\eta
$$

If $|\zeta|$ is small $\left(|\zeta| \leq \frac{\varepsilon}{2 R_{0}}\right.$, say), then elementary Taylor expansion gives us the bound

$$
\left|\tau_{z, \zeta}(1)^{-1} d \exp _{z}(\zeta)(h, \eta)-(h+\eta)\right| \leq(1+O(\varepsilon))\left(c_{1} \varepsilon^{2}|h|+c_{2} \varepsilon^{2}|\eta|\right) .
$$

Thus there exists some uniform $\varepsilon_{0}$ such that in the balls $|\zeta| \leq \frac{\varepsilon}{\sqrt{2} R_{0}}$ for any $\varepsilon \leq \varepsilon_{0}$ the differential is a diffeomorphism and is even $O\left(\varepsilon^{2}\right)$ close to the identity.

Remark 6.1. Similar estimates can be obtained in the Hermitian case either, geodesics being defined by the Chern connection rather than the Levi-Civita connection. One then has to assume additionally a uniform bound on $|\partial \omega|_{\omega}$ and $|D(\partial \omega)|_{\omega}$ to accommodate the presence of torsion. However, replacing exp by exph as was done in [D4] and [BD] would be a challenge, because we would then need an "effective" version of E. Borel's theorem to show that exph can be chosen to satisfy the same estimates as exp, and this is certainly non trivial.

Acknowledgements. This project was initiated during the BIRS conference "Complex MongeAmpère equation" held in 2009. The authors would like to acknowledge the perfect working conditions provided by the organizers. Part of the research was done while the second and fifth authors were visiting the Erwin Schrödinger institute in Vienna in 2009. They would like to express their gratitude for the hospitality. The second author was supported by Polish ministerial grant "Iuventus Plus" and Kuratowski fellowship granted by the Polish Mathematical Society (PTM) and Polish Academy of Science (PAN). The research was done while the fourth author was a postdoctoral research fellow at Centro Internazionale per la Ricerca Matematica, Trento, Italy. He would like to thank the members of the Institute for their kind hospitality. The second and fifth authors were partially supported by NCN grant 2011/01/B/ST1/00879.

\section{REFERENCES}

[]BCHM C. Birkar, P. Cascini, C. Hacon and J. McKernan, Existence of minimal models for varieties of log general type, J. Amer. Math. Soc. 23 (2010), no. 2, 405-468.

[]BEGZ S. Boucksom, P. Eyssidieux, V. Guedj and A. Zeriahi, Monge-Ampère equations in big cohomology classes, Acta Math. 205 (2010), 199-262.

[]BGZ S. Benelkourchi, V. Guedj and A. Zeriahi, A priori estimates for weak solutions of complex MongeAmpère equations, Ann. Sc. Norm. Super. Pisa Cl. Sci. (5) 7 (2008), no. 1, 81-96.

[]BT1 E. Bedford and B. A. Taylor, The Dirichlet problem for the complex Monge-Ampère operator, Invent. Math. 37 (1976), 1-44.

[]BT2 E. Bedford and B. A. Taylor, A new capacity for plurisubharmonic functions, Acta Math. 149 (1982), $1-40$.

[]Ber R. Berman, A thermodynamical formalism for Monge-Ampère equations, Moser-Trudniger inequalities and Kähler-Einstein metrics, Preprint arXiv:1011.3976v1.

[]BD R. Berman and J.-P. Demailly, Regularity of plurisubharmonic upper envelopes in big cohomology classes, preprint arXiv 0905.1246v1, to appear in the Proceedings of the volume "Geometry and Topology" in honor of Oleg Y. Viro, edited by B. Juhl-Jöricke and M. Passare, Birkhaüser. 
[]D1 J.-P. Demailly, Estimations $L^{2}$ pour l'opérateur $\bar{\partial}$ d'un fibré vectoriel holomorphe semi-positif au-dessus d'une variété kählérienne complète, Ann. Sci. École Norm. Sup. 4e Sér. 15 (1982), 457-511.

[]D2 J.-P. Demailly, Regularization of closed positive currents and intersection theory, J. Alg. Geom. 1 (1992), 361-409.

[]D3 J.-P. Demailly, Monge-Ampère operators, Lelong numbers and intersection theory, Complex analysis and geometry, 115-193, Univ. Ser. Math., Plenum, New York (1993).

[]D4 J.-P. Demailly, Regularization of closed positive currents of type (1,1) by the flow of a Chern connection, Aspects of Math. Vol. E26, Vieweg (1994), 105-126.

[]DP J.-P. Demailly and N. Pali, Degenerate complex Monge-Ampère equations over compact Kähler manifolds, Int. J. Math. 21 (2010), 357-405.

[]DNS T. C. Dinh, V. A. Nguyen and N. Sibony, Exponential estimates for plurisubharmonic functions and stochastic dynamics, Journal Diff. Geom. 84 (2010), 465-488.

[]DS T. C. Dinh and N. Sibony, Distribution des valeurs de transformations meromorphes et applications, Comment. Math. Helv. 81 (2006), 221-258.

[]Di S. Dinew, Hölder continuous potentials on manifolds with partially positive curvature, J. Inst. Math. Jussieu 9 (2010), 705-718.

[]DZ S. Dinew and Z. Zhang, On stability and continuity of bounded solutions of degenerate complex MongeAmpère equations over compact Kähler manifolds, Adv. Math. 225 (2010), 367-388.

[]EGZ1 P. Eyssidieux, V. Guedj and A. Zeriahi, Singular Kähler-Einstein metrics, J. Amer. Math. Soc. 22 (2009), 607-639.

[]EGZ2 P. Eyssidieux, V. Guedj and A. Zeriahi, A priori $L^{\infty}$-estimates for degenerate complex Monge-Ampère equations, Int. Math. Res. Not. (2008), ID rnn070.

[]EGZ3 P. Eyssidieux, V. Guedj and A. Zeriahi, Viscosity solutions to degenerate complex Monge-Ampère equations, Comm. Pure Appl. Math. 64 (2011), 1059-1094.

[]GT D. Gilbarg and N. Trudinger, Elliptic partial differential equations of second order, Grundl. der Math. Wiss. Springer Verlag, 244 (1983).

[]GKZ V. Guedj, S. Kołodziej and A. Zeriahi, Hölder continuous solutions to the complex Monge-Ampère equations equations, Bull. London Math. Soc. 40 (2008), 1070-1080.

[]GZ1 V. Guedj and A. Zeriahi, Intrinsic capacities on compact Kähler manifolds, J. Geom. Anal. 15 (2005), no.4, 607-639.

[]GZ2 V. Guedj and A. Zeriahi, Stability of solutions to Monge-Ampère equations in big cohomology classes, preprint 2011

[]Hi P. H. Hiep, Hölder continuity of solutions to the complex Monge-Ampère equations on compact Kähler manifolds, Ann. Inst. Fourier 60 (2010), 1857-1869.

[]HK E. Heintze and H. Karcher, A general comparison theorem with applications to volume estimates for submanifolds, Ann. Sci. Éc. Norm. Sup. 11 (1978) 451-470

[]Ki1 C. O. Kiselman, Partial Legendre transformation for plurisubharmonic functions, Invent. Math. 49 (1978), 137-148.

[]Ki2 C. O. Kiselman, Attenuating the singularities of plurisubharmonic functions, Ann. Polon. Math. 60 (1994), 173-197.

[]K1 S. Kołodziej, The complex Monge-Ampère equation, Acta Math. 180 (1998), 69-117.

[]K2 S. Kołodziej, The Monge-Ampère equation on compact Kähler manifolds, Indiana Univ. Math. J. 52 (2003), no. 3, 667-686.

[]K3 S. Kołodziej, Hölder continuity of solutions to the complex Monge-Ampère equation with the right hand side in $L^{p}$. The case of compact Kähler manifolds, Math. Ann. 342 (2008), 379-386.

[]KT S. Kołodziej and G. Tian, A uniform $L^{\infty}$ estimate for complex Monge-Ampère equations, Math. Ann. 342 (2008), 773-787.

[]Pl S. Pliś, A counterexample to the regularity of the degenerate complex Monge-Ampère equation, Ann. Polon. Math. 86 (2005), 171-175.

[]Sk H. Skoda, Sous-ensembles analytiques d'ordre fini ou infini dans $\mathbb{C}^{n}$, Bull. Soc. Math. France 100 (1972), 353-408.

[]ST1 J. Song and G. Tian, The Kähler-Ricci flow on surfaces of positive Kodaira dimension, Invent. Math. 170 (2007), 609-653.

[]ST2 J. Song and G. Tian, Canonical measures and Kähler-Ricci flow, preprint arXiv 0802.2570v1.

[]ST3 J. Song and G. Tian, The Kähler-Ricci flow through singularities, preprint arXiv 0909.4898v1.

[]SW J. Song and B. Weinkove, Contracting exceptional divisors by the Kähler-Ricci flow, Preprint arXiv:1003.0718v1.

[]TZ G. Tian and Z. Zhang, On the Kähler-Ricci flow on projective manifolds of general type, Chinese Ann. Math. B 27 (2) (2006), 179-192. 
[]To V. Tosatti, Limits of Calabi-Yau metrics when the Kähler class degenerates, J. Europ. Math. Soc, 11(2009), 755-776.

[]Ze A. Zeriahi, Volume and capacity of sublevel sets of a Lelong class of plurisubharmonic functions, Indiana Univ. Math. J. 50 (2001), no. 1, 671-703.

[]Z Z. Zhang, On degenerate Monge-Ampère equations over closed Kähler manifolds, Int. Math. Res. Not. 11 (2006) ID 63640, 18p.

Jean-Pierre Demailly: Institut Universitaire de France et Université Grenoble I, 100 rue des Maths 38402 Saint-Martin d'Hères, France;

e-mail: demailly@fourier.ujf-grenoble.fr

Sławomir Dinew: Rutgers University, Newark, NJ 07102, USA;

Jagiellonian University 30-348 Krakow, Lojasiewicza 6, Poland;

e-mail: slawomir.dinew@im.uj.edu.pl

Vincent Guedj: Institut Universitaire de France et Institut de Mathématiques de Toulouse, Université Paul Sabatier, 31602 Toulouse Cedex 09, France;

e-mail: vincent.guedj@math.univ-toulouse.fr

Pham Hoang Hiep: Hanoi National University of Education, Tuliem-Hanoi-Vietnam;

e-mail: phhiep_vn@yahoo.com

Sławomir Kołodziej: Jagiellonian University 30-348 Krakow, Lojasiewicza 6, Poland;

e-mail: slawomir.kolodziej@im.uj.edu.pl

Ahmed Zeriahi: Institut de Mathématiques de Toulouse, Université Paul Sabatier, 31602 Toulouse Cedex 09, France;

e-mail: ahmed.zeriahi@math.univ-toulouse.fr 